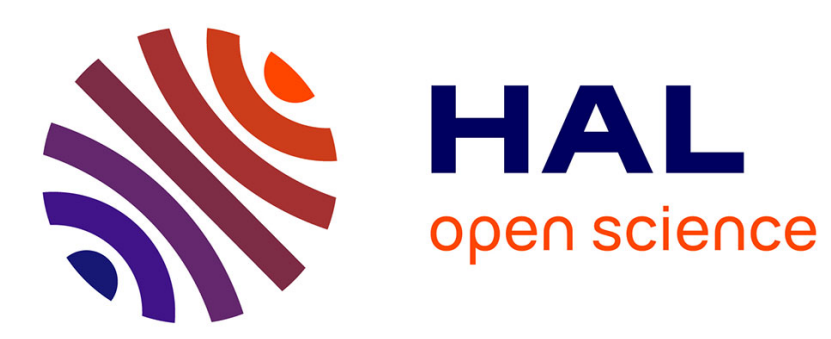

\title{
Le programme archéologique franco-indonésien sur Padang Lawas (Sumatra Nord). Réflexions préliminaires
}

Daniel Perret, Heddy Surachman, Lucas Partanda Koestoro, Sukawati Susetyo

\section{To cite this version:}

Daniel Perret, Heddy Surachman, Lucas Partanda Koestoro, Sukawati Susetyo. Le programme archéologique franco-indonésien sur Padang Lawas (Sumatra Nord). Réflexions préliminaires. Archipel, 2007, 74, pp.45-82. 10.3406/arch.2007.3915 . halshs-01885203

\section{HAL Id: halshs-01885203 \\ https://shs.hal.science/halshs-01885203}

Submitted on 1 Oct 2018

HAL is a multi-disciplinary open access archive for the deposit and dissemination of scientific research documents, whether they are published or not. The documents may come from teaching and research institutions in France or abroad, or from public or private research centers.
L'archive ouverte pluridisciplinaire HAL, est destinée au dépôt et à la diffusion de documents scientifiques de niveau recherche, publiés ou non, émanant des établissements d'enseignement et de recherche français ou étrangers, des laboratoires publics ou privés. 


\title{
Le programme archéologique franco-indonésien sur Padang
} Lawas (Sumatra Nord). Réflexions préliminaires

\author{
Daniel Perret, Heddy Surachman, Lucas Partanda Koestoro, Sukawati Susetyo
}

\author{
Abstract \\ Daniel Perret, École française d'Extrême-Orient, Paris. Lucas P. Koestoro, Balai Arkeologi, Medan. Heddy Surachman \& \\ Sukawati Susetyo, Pusat Penelitian dan Pengembangan Arkeologi Nasional Indonesia, Jakarta \\ Le programme archéologique franco-indonésien sur Padang Lawas (Sumatra Nord). Réflexions préliminaires
}

The Padang Lawas area in the North Sumatra Province has been known since the middle of the 19th century for its numerous Hindu-Buddhist remains located on the upper course of the Barumun river basin, at the foot of the Barisan range. This paper begins with a brief review of the research on architecture, art and inscriptions conducted in Padang Lawas during the last century. Suggested datings for the temples by previous authors range from the 11th to the 14th century and some indications point to the practice of Vajrayåna Buddhism, Sivaism and a Buddhist-Sivaist syncretism. By contrast, researches on ancient settlements in Padang Lawas started much more recently, less than ten years ago in fact, with the rediscovery of a complex system of earthen walls and moats at Si Pamutung. The promising results of the first test-pits did encourage the launching in 2006 of the second phase of a joint French-Indonesian archaeological program focused on the ancient settlements of Tapanuli since 2001. Pottery, Chinese ceramics, glass, beads and terra-cottas, as well as the remains of a brick structure were found during this first campaign. These finds confirm that Si Pamutung was a major settlement in Padang Lawas. These first results raise questions on the chronology, the nature and the structure of the Si Pamutung settlement itself. In addition, their combination with previous data brings new hypotheses regarding the population of Padang Lawas, its connection with coastal settlements in the region and its relation with neighbouring economic and political power, as well as farther centres in Asia.

\section{Citer ce document / Cite this document :}

Perret Daniel, Surachman Heddy, Partanda Koestoro Lucas, Susetyo Sukawati. Le programme archéologique francoindonésien sur Padang Lawas (Sumatra Nord). Réflexions préliminaires. In: Archipel, volume 74, 2007. pp. 45-82;

doi : 10.3406/arch.2007.3915

http://www.persee.fr/doc/arch_0044-8613_2007_num_74_1_3915

Document généré le 16/12/2016 


\section{DANIEL PERRET, HedDy SURACHMAN, LUCAS P. KoESTORO, SUKAWATI SUSETYO}

\section{Le programme archéologique franco-indonésien sur Padang Lawas (Sumatra Nord). Réflexions préliminaires}

À Sumatra Nord, les premières véritables recherches archéologiques sur les sites d'habitat ancien d'époque historique remontent au début des années 1970 avec les fouilles menées à Kota Cina, un site sur la côte est, entre Medan et Belawan, occupé entre la fin du $\mathrm{XI}^{\mathrm{e}}$ siècle et la première moitié du $\mathrm{XIV}^{\mathrm{e}}$ siècle ${ }^{1}$. Une dizaine d'années plus tard, des archéologues du Centre National de la Recherche Archéologique d'Indonésie entreprenaient les premiers sondages à Barus, sur la côte opposée. Cette initiative déboucha sur la mise en place, en 1995, d'un programme de recherche franco-indonésien centré sur le site côtier de Lobu Tua. Ce programme fut achevé en 2000, après cinq campagnes de fouilles intensives sur cette ville occupée entre le milieu du IXe et la fin du $\mathrm{XI}^{\mathrm{e}}$ siècle $^{2}$. Un nouveau programme de coopération entre l'École française d'Extrême-Orient et le Centre National de la Recherche Archéologique d'Indonésie ${ }^{3}$ se mit en place, dès l'année suivante, avec pour objectif les sites urbains de la région de Tapanuli, qui couvre en gros la moitié sud de la province. La première phase, entre 2001 et 2005,

1. McKinnon, 1984 et 2003.

2. Guillot (éd.), 1998; Guillot, Surachman, Perret et al., 2003.

3. Ces deux programmes de recherche archéologique en coopération ont bénéficié et bénéficient du soutien de la commission consultative des recherches archéologiques à l'étranger du Ministère des Affaires Étrangères français. 
s'intéressa aux autres sites d'habitat ancien de la région de Barus, en particulier à Bukit Hasang, occupé dès le XII e siècle ${ }^{4}$. C'est en se basant sur les connaissances acquises au cours des onze années consacrées à la côte ouest, que la seconde phase du programme, qui débuta en 2006, s'intéressa, cette fois, aux sites de l'intérieur de l'île. C'est Padang Lawas, région bien connue pour son ensemble de temples hindo-bouddhiques dans l'actuel kabupaten de Tapanuli Selatan, qui a été choisie.

Padang Lawas est un cas d'école dans l'historiographie du monde malais. Dès sa découverte, et pendant un siècle et demi, les recherches y furent consacrées uniquement aux monuments, à la sculpture, aux objets en bronze et à l'épigraphie. La présence des sanctuaires focalisa tellement l'attention des chercheurs, que l'intérêt pour l'habitat s'éveilla encore plus tardivement qu'à Kota Cina et à Barus. Il remonte en effet à moins de dix ans. La richesse archéologique exceptionnelle de cette zone, à l'échelle de Sumatra, offre l'opportunité d'éclairer, d'une part, sa propre histoire, d'autre part, les articulations de nature économique, politique, religieuse et culturelle non seulement avec les sites des côtes occidentale et orientale, mais également avec d'autres régions de l'intérieur de Sumatra, du monde malais et de l'Asie en général.

\section{Données géographiques}

Padang Lawas, ou plus exactement Padang Bolak «vaste terre» en langue locale, peut se définir de trois manières différentes. Si l'on s'en tient à sa signification première, il s'agit d'une basse vallée (altitude inférieure à $100 \mathrm{~m}$ ), limitée à l'est par les villes ou villages de Gunung Tua, Binanga et Aeknabara, qui s'enfonce comme un coin dans les contreforts orientaux de la chaîne des Barisan. Administrativement, elle correspond aujourd'hui aux districts (kecamatan) de Padang Bolak et Padang Bolak Julu ${ }^{5}$. Les historiens et les archéologues lui confèrent une autre dimension à partir des vestiges disséminés dans le bassin du fleuve Barumun, en amont de la petite ville de Binanga. Le fleuve, qui prend sa source à environ mille mètres d'altitude dans les montagnes entre Sibuhuan et Siabu, est encore ici à quelque 200 kilomètres de son embouchure dans le détroit de Malacca. Si la plupart des vestiges occupent effectivement la partie basse, quelques-uns sont perchés dans les premiers contreforts des Bukit Barisan, à une altitude dépassant 300 mètres. Autrefois navigable par de petites embarcations au moins jusqu'à Binanga, au confluent avec la Batang Pane, le cours supérieur de la Barumun

4. Perret \& Surachman (éd.), à paraître.

5. La région n'échappe pas au phénomène de redécoupages administratifs qui fait suite à la mise en place des lois sur l'autonomie régionale. Ainsi, un kabupaten de Padang Lawas, centré sur Gunung Tua, verra le jour en 2007. 
ne l'est plus aujourd'hui pour cause d'envasement et de captages pour l'irrigation 6 . On est par conséquent très loin de l'animation encore visible loin dans l'intérieur des terres sur des rivières plus importantes de Sumatra comme la Siak ou la Musi.

La basse plaine et les premiers contreforts offrent un paysage le plus souvent désolé, proche de la steppe, voire du semi-désert dans les collines du nord. Cet environnement particulier est généralement attribué à un vent de type foehn qui, en soufflant de façon continue durant plusieurs mois de l'année, assèche les sols et peut rendre impossible la mise en œuvre de la plupart des cultures. D'autres raisons ont été avancées pour expliquer ce paysage, nous y reviendrons dans la suite. Ce vent sec est probablement lié, au moins en partie, à l'existence d'un couloir qui traverse la chaîne de montagnes dans le prolongement de Padang Lawas, et débouche sur la côte ouest, un peu au sud de Sibolga. Aujourd'hui emprunté par la route qui relie cette ville côtière à Padangsidempuan, ce passage, d'une altitude maximale inférieure à 400 mètres, est certainement l'un des plus faciles sur l'ensemble de la chaîne des Barisan.

\section{L'architecture, l'art et les inscriptions de Padang Lawas}

\section{Architecture et sculpture}

À ce jour, dix-neuf sites comportant soit un complexe d'édifices, soit un édifice isolé, ont été repérés (voir carte des sites) ${ }^{7}$. Dix-sept sont encore visibles aujourd'hui : Sangkilon, Si Pamutung, Tandihat I, Tandihat II, Tandihat III, Bahal I, Bahal II, Bahal III, Pulo, Bara, Haloban, Sitopayan, Nagasaribu, Mangaledang, Pageranbira, Tanjungbangun et Aektunjang ${ }^{8}$. Appelés localement biaro $^{9}$, tous ces sites, sauf un, se trouvent en amont du confluent de la Barumun et de la Batang Pane, plus précisemment le long de ces cours d'eaux et de la rivière Sirumambe, un affluent de la Batang Pane. L'architecture allie généralement la brique et des éléments décoratifs en tuf, mais plusieurs vestiges de structures uniquement en pierre sont également

6. En 1920, le Résident de Tapanuli note dans son journal que la limite de navigabilité a reculé de quinze kilomètres en un quart de siècle (Callenfels, $1920: 72$ ).

7. Je remercie Mr. Yanto Wahyantono (IRD Jakarta) pour la numérisation des cartes et du plan.

8. Le sanctuaire de Gunung Tua n'existait déjà plus à l'époque coloniale. L'autre site disparu est celui de Porlak Dolok (près du village de Paringgonan).

9. Terme dérivé du sanskrit wihāra que l'on traduit généralement par «monastère». Ces édifices n'ayant pu servir de monastères, Damais suggère que les monastères qui étaient attachés à chacun d'eux étaient construits en matériaux périssables et ont donc disparu depuis. Le terme serait resté en usage pour désigner les seuls monuments en dur (Damais, 1963 : note 3, p. 541). 


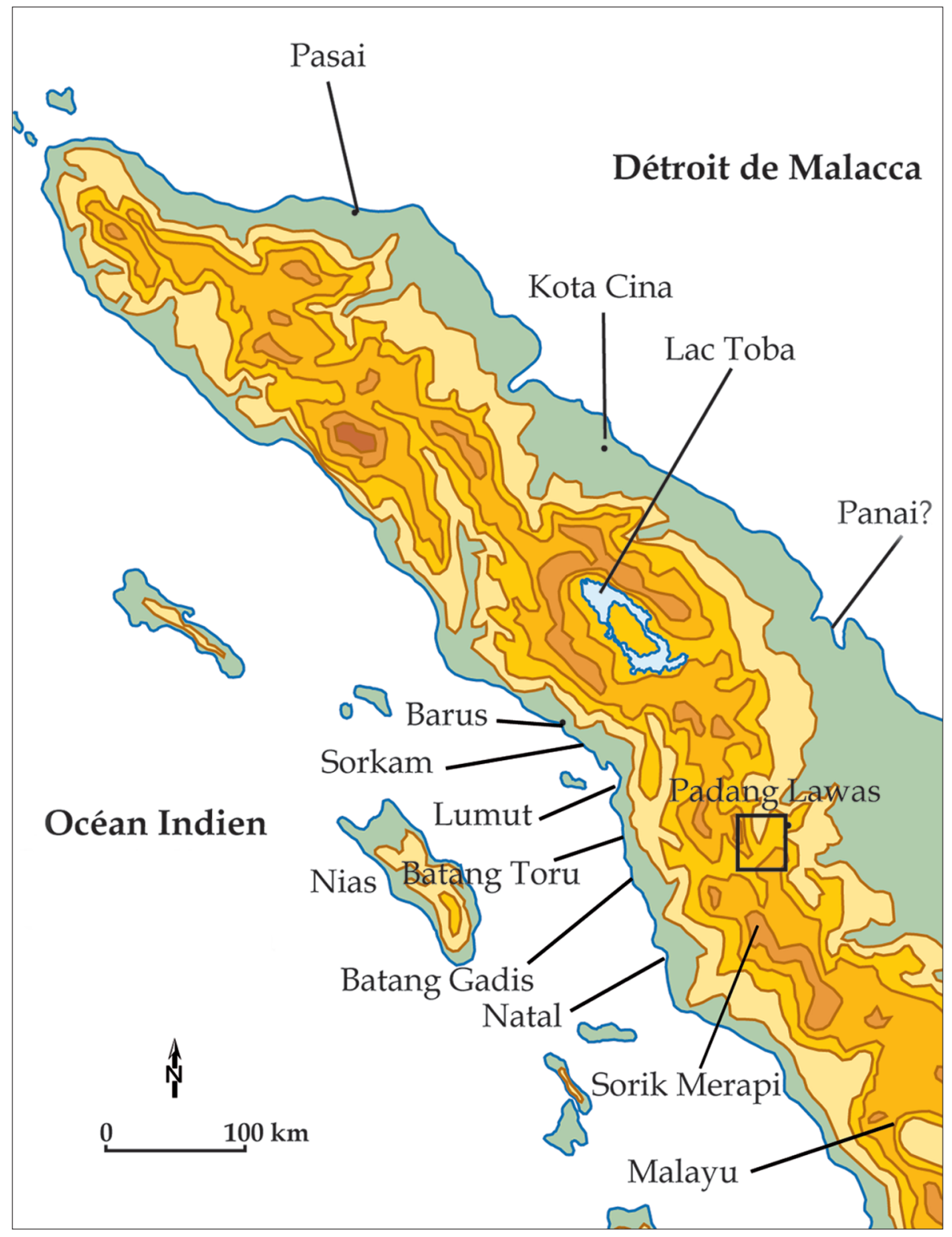

Principaux toponymes cités 


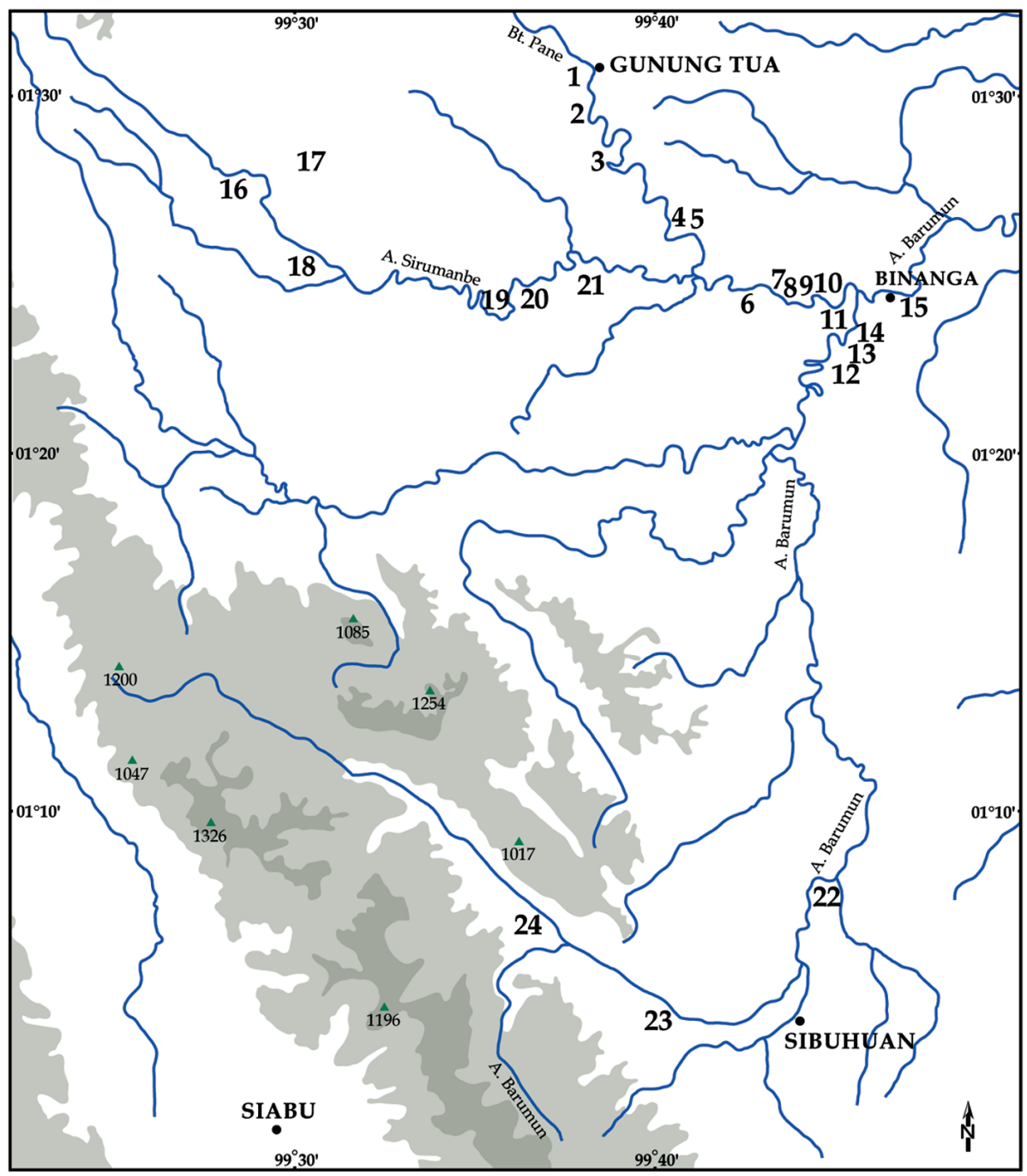

Altitude (en mètres)

$500-1000 \quad 1000$ et plus

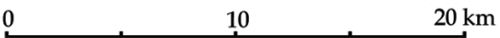

Sites archéologiques de Padang Lawas

1. Gunung Tua, 2. Sitopayan, 3. Hayuara, 4. Tanjung Bangun, 5. Haloban, 6. Bara, 7. Pulo, 8. Bahal I, 9. Bahal II, 10. Bahal III, 11. Si Pamutung, 12. Tandihat I, 13. Tandihat II, 14. Tandihat III, 15. Aek Tunjang, 16. Batu Gana, 17. Sisoldop, 18. Padang Bujur, 19. Nagasaribu, 20. Sihodahoda, 21. Mangaledang, 22. Sangkilon, 23. Porlak Dolok, 24. Pageranbira. 
visibles. À cette liste, il faut ajouter cinq sites ayant livré uniquement des pierres sculptées : Hayuara, Batu Gana, Sihodahoda, Sisoldop et Padangbujur ${ }^{10}$. Seul Sisoldop, au sommet d'une colline, est éloigné d'un cours d'eau.

Sans entrer dans le détail, nous donnerons ici les caractéristiques générales de la plupart des complexes d'édifices, d'après les quelques sites entièrement dégagés à ce jour. Ces ensembles sont limités par un mur d'enceinte en briques, de taille variable. Le sanctuaire principal, en briques, s'ouvre à l'est. Implanté dans la moitié ouest de la cour, il est légèrement décalé vers le sud par rapport à l'axe de la porte unique de l'enceinte ouverte à l'est. La cella, généralement carrée, repose sur un ou deux soubassements également carrés (Si Pamutung (photo 1), Bahal I, Sangkilon). Elle est prolongée par une superstructure ronde, carrée ou octogonale. Des traces de plâtre ou de stuc ont été relevées sur les murs intérieurs des cellas de Sangkilon, Tandihet I, Pamutung, Bahal II, III et Bara, ainsi que sur les murs extérieurs de Bahal III ${ }^{11}$. Une terrasse-pendopo carrée, généralement en briques, est implantée devant le bâtiment principal. Des terrasses en nombre variable, le plus souvent en briques, flanquent ces deux édifices au nord et au sud. Un tuf local 12 a été utilisé dans la construction d'au moins une de ces terrasses (photo 2) et on le retrouve employé également pour certains ornements architecturaux. Ce matériau est par ailleurs omniprésent dans la sculpture : makara à la base des escaliers, lions assis, raksasa, stambha, piédestals, divinités. Les sites de Padang Lawas ont par ailleurs livré un certain nombre d'objets en bronze sur lesquels nous reviendrons.

Tout commença pour la recherche lorsque, peu après la conquête de Padang Lawas par les Hollandais, Franz Junghuhn attira l'attention sur les vestiges en 1846. Rosenberg en livra les premières représentations dix ans plus tard ${ }^{13}$. Callenfels fut le premier scientifique à se rendre sur place, en 1920. Il visita sept sites et lança le dégagement des complexes de Sitopayan et de Bahal $\mathrm{I}^{14}$. Bosch traversa la région dix ans plus tard et, comme

10. Nous n'incluons pas dans ce décompte le site de Lobu Dolok (Desa Aek Tolong Tonga, kec. Padang Bolak). Il s'agit de monuments funéraires faits d'alignements de dalles ou de blocs de pierre comportant des inscriptions en langue locale, qui appartiennent, selon nous, à une période plus récente. Le même genre de monument comportant des inscriptions a été repéré à Padangbujur et à Batu Gana, mais là ils côtoient des vestiges que nous considérons comme plus anciens.

11. Schnitger, 1937a : 34. Suleiman observera de nouveau ces traces de plâtre à Bahal III, quarante ans plus tard (1983: 17).

12. Une carrière de tuf est toujours en exploitation à Paranginan, près de Gunung Tua. Ce matériau est employé aujourd'hui pour renforcer les fondations des constructions.

13. Rosenberg, 1855.

14. Callenfels, 1920, 1925 ; Haan, 1926. 


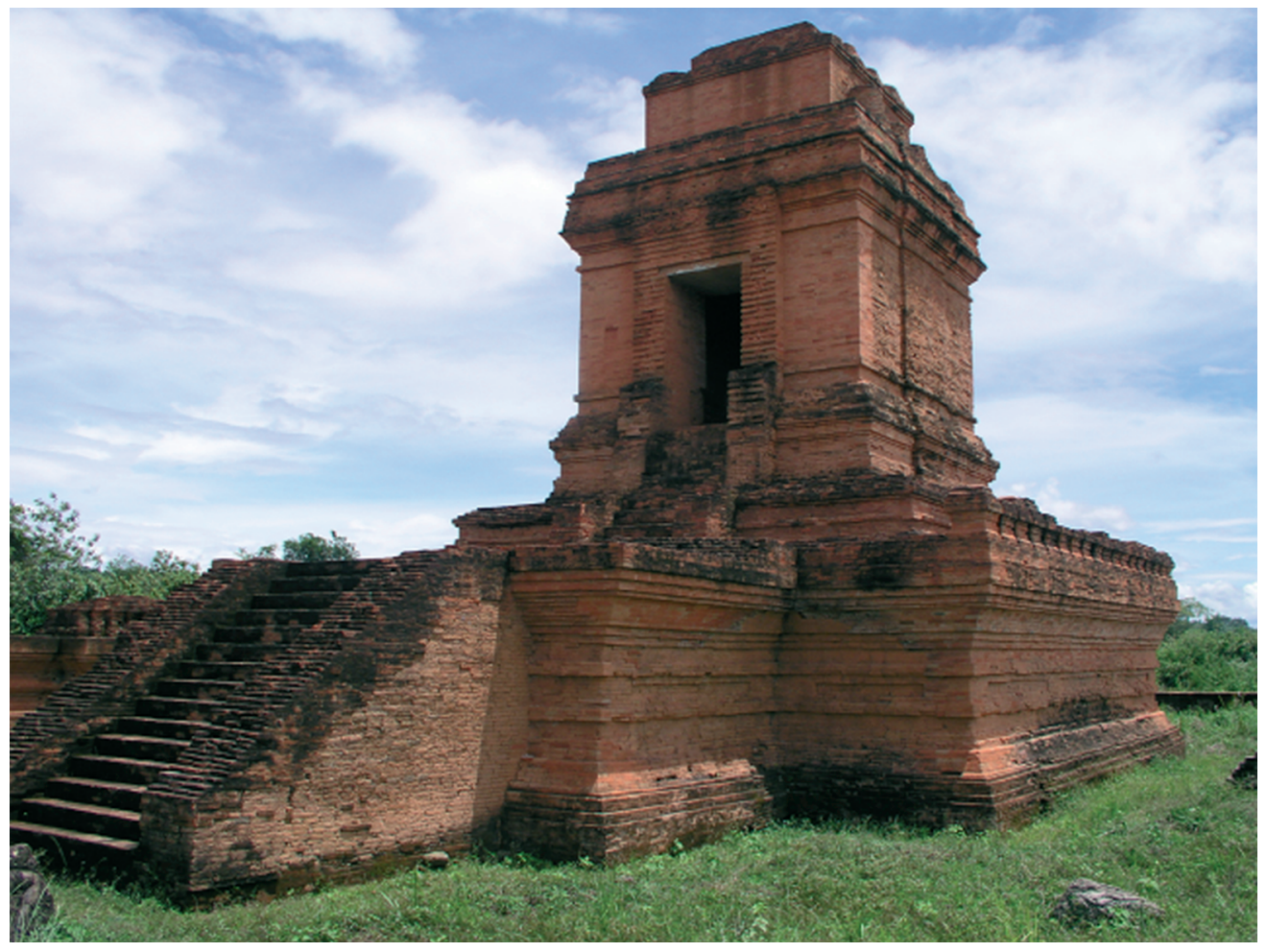

1. Si Pamutung : le sanctuaire principal après restauration 


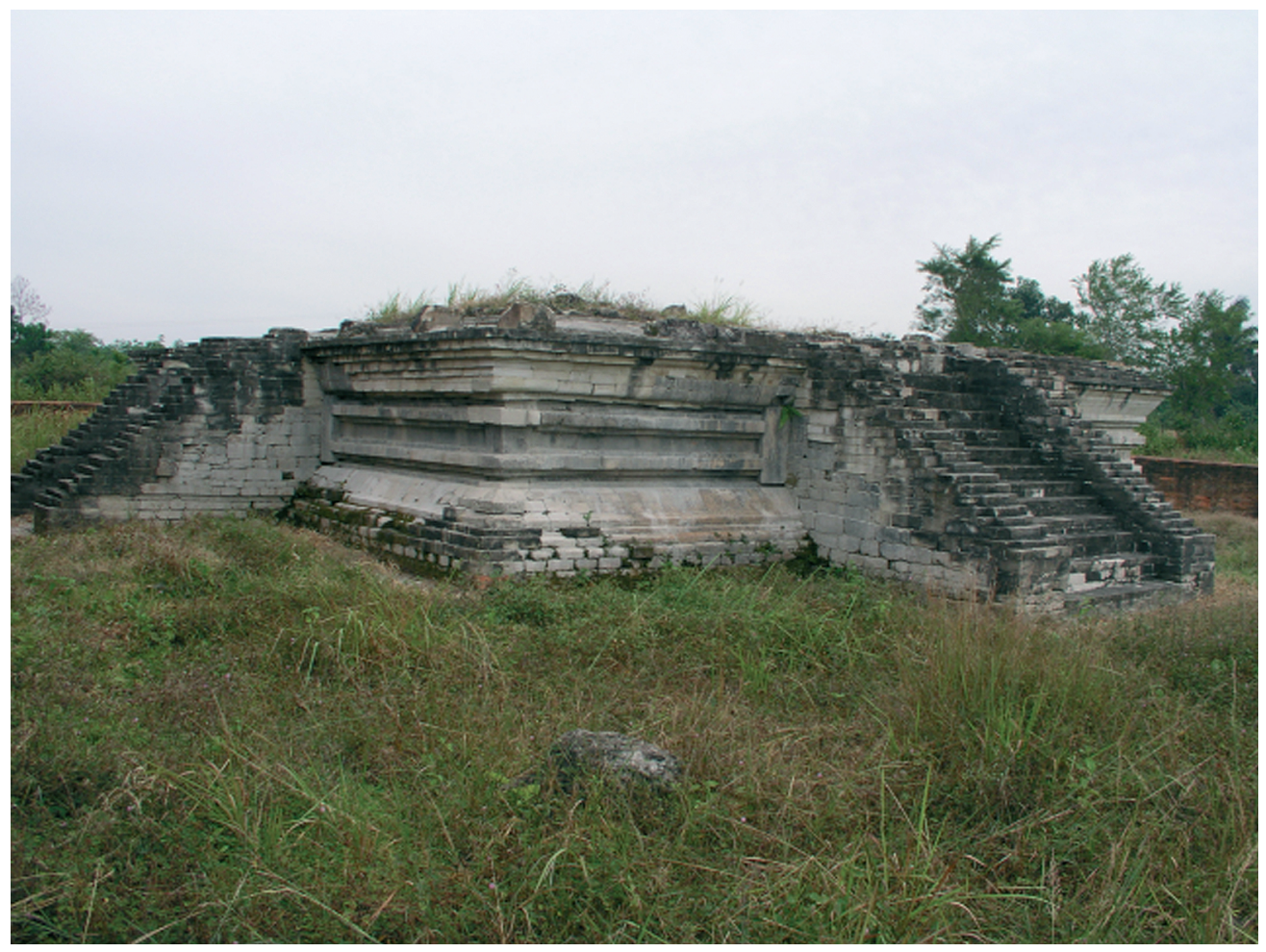

2. Si Pamutung : terrasse en tuf en cours de restauration 
Callenfels, ne visita qu'une partie des sites ${ }^{15}$. Il fut suivi par Schnitger, alors conservateur du musée de Palembang, qui commença ses prospections et dégagements à Padang Lawas en 1935. Si, avec un recul de 70 ans, les méthodes et certaines des hypothèses de Schnitger apparaissent criticables, on lui doit néanmoins la liste déjà pratiquement complète des sites de la région et ses données sur l'architecture et la sculpture seront largement diffusées. L'insuffisance des ressources humaines et financières firent que les quarante années suivantes furent jalonnées uniquement par quelques visites de spécialistes.

C'est en effet seulement à la fin des années 1970 que les travaux reprennent à Padang Lawas, cette fois dans le cadre d'un programme de restauration du complexe de Bahal I par le Service des Antiquités ${ }^{16}$. Ces interventions vont s'enchaîner jusqu'à aujourd'hui avec les restaurations successives de Bahal II, Bahal III et Si Pamutung. Les fouilles archéologiques reprennent quant à elles à partir de 1994 avec des dégagements partiels de structures dans les complexes de Tandihet II ${ }^{17}$ et de Bara ${ }^{18}$.

\section{Les inscriptions}

À ce jour, dix inscriptions anciennes ont été découvertes et déchiffrées partiellement ou totalement ${ }^{19}$. Ce sont :

- un ensemble en bronze comprenant trois personnages à l'origine (dont l'un a disparu), trouvé dans les années $1880 \mathrm{chez}$ le raja de Gunung Tua où il était conservé comme pusaka. Le personnage central est Lokanātha, alors que le personnage secondaire est Tārā assise. Les trois lignes sur le socle sont en vieux-malais et en sanskrit, notés en caractères «vieux-javanais». L'inscription contient en particulier un nom, Suryya, le fabricant de l'objet, et une date lue «30 mars 1039» (961 Śaka) par Damais. L'emploi du mot malais barbwat indique qu'il s'agit très probablement d'un objet originaire de Sumatra ${ }^{20}$. Bien que trouvé hors contexte, la qualité de son ancien détenteur permet de penser qu'il appartient à l'histoire de Padang Lawas. Par contre, rien ne prouve qu'il provienne précisemment du sanctuaire aujourd'hui disparu de Gunung Tua.

15. Bosch, 1930.

16. Departemen Pendidikan dan Kebudayaan, 1996 : 39.

17. Susanto et al., 1994; Utomo et al., 1995 ; Utomo, 1996.

18. Susanto et al., 1995, 1997a, 1997b, 1997c.

19. Un autre support apparemment inscrit et peut-être ancien est signalé par le contrôleur James en 1894. Il s'agirait d'une statue «batak» de Pintu Bosi, à «la limite de Padang Lawas» (NBG, 37, 1899:12).

20. Coll. Musée National de Jakarta, no. B626d (NBG, 25, 1887 : 175-6, 185; NBG, 26, 1888 : 60; Kern, 1917 : 142-144; Krom, 1931 : 247; Damais, 1952 : 100-101; 1955 : 207-208). 
- une pierre inscrite de deux lignes, collectée par Schnitger dans le complexe de Tandihet I. Les caractères sont en vieux-javanais, et la langue en vieuxmalais. L'inscription est accompagnée d'une représentation de crâne et de croissant de lune (candra kapala). Il s'agit de la partie introductive d'un texte qui livre une date lue «26 avril 1179» (1101 Saka) par Damais ${ }^{21}$.

- un pilier en pierre au sommet orné d'une tête de Ganeśa. Repéré par Callenfels au lieu-dit Porlak Dolok, près du village de Paringgonan, à quelques kilomètres de la source de la Barumun. Il s'agit d'une inscription bilingue comprenant, dans la partie supérieure, six lignes en vieux-malais utilisant des caractères vieux-javanais et, dans la partie inférieure, sept lignes en tamoul. Les textes semblent avoir le même contenu : un certain «senāpati rakandi pankamendāpatyarāmirā» (Sedyawati) ou «senāpati rakan dīpañkara» (Karashima) fait une offrande pour le mérite du roi nommé «Pāduka Śrī Mahārāja» dans l'inscription javanaise, «Peritu Śrī Mahārāja» dans celle en tamoule. Les textes comportent une date dont la lecture est loin de faire l'unanimité : 1213 EC (1135 Śaka) pour Damais, 1245 EC (1167 Śaka) pour Callenfels et 1265 EC (1187 Śaka) pour Karashima 22.

- un piédestal en tuf provenant du complexe de Sitopayan. L'inscription comprend trois lignes réparties sur deux faces. Il s'agit d'un texte en vieuxmalais utilisant des caractères vieux-batak (Casparis), qui indique la construction d'un sanctuaire par plusieurs individus dont les noms n'apparaissent pas toujours clairement : «hang Tahi si Rangngit kabayin (?) et (?) pu Anyawārin» (d'après Bosch) ${ }^{23}$.

- un piédestal en tuf provenant du complexe de Sitopayan. L'inscription comprend deux lignes. Il s'agit d'un texte en vieux-malais utilisant des caractères vieux-batak (Casparis), indiquant la construction d'un monument par «pu Sapta hang Buddhi sang Imba et (?) hang Langgar [en souvenir] de la construction d'un wihāra pour Sa Majesté le Roi» (Bosch) ${ }^{24}$.

- une plaquette rectangulaire en or collectée par Schnitger à Tandihet II. L'inscription de huit lignes en caractères năgarí comporte une représentation de vajra. La langue est un mélange d'onomatopées et de sanskrit. Les diffé-

21. Coll. Musée National de Jakarta, no. D.165 (Schnitger, 1936a : 13, note 1, p. 38; 1937 : 21; JBGKW, III, $1936: 199$; Damais, 1952:100-101; $1955: 208)$.

22. Coll. Musée National de Jakarta, no. D.181 (Callenfels, 1920 : 70; Bosch, 1930 : 147; Krom, 1931 : 304; Damais, 1952:100-101; $1955: 208-209$; Sedyawati, $1994: 556$; Karashima, 2002: 14).

23. Coll. musée provincial de Medan, no. 1517.1 (Callenfells, 1920 : 66; Bosch, 1930 : 135; Nik Hassan Shuhaimi, 1992 : 77; Setianingsih, $2001: 95$ ).

24. Coll. musée provincial de Medan, no. 1517.2 (Bosch, 1930 : 135; Nik Hassan Shuhaimi, 1992 : 77; Setianingsih, 2001 : 95). Goris a suggéré de lire le chronogramme 1157 S (1235 EC) dans cette inscription (Schnitger, 1937a : 32), hypothèse réfutée par Damais (1963 : note 3 , p. 542). 
rents sons feraient référence au rire rituel accompli durant certaines cérémonies tantriques. Elle est datée paléographiquement de fin XIIIe-XIVe siècle ${ }^{25}$.

- une plaquette rectangulaire en or collectée par Schnitger à Sangkilon. L'inscription de huit lignes en caractères nāgarī a pour langue le sanskrit et comporte une représentation de vajra. Le contenu, à caractère religieux, aurait un rapport avec la consécration d'une statue de Yamari ${ }^{26}$.

- Un cylindre de pierre grise feuilletée collecté à Bahal I (probablement lors des travaux de restauration). Le texte de dix lignes est en vieux-malais utilisant des caractères vieux-javanais. Daté paléographiquement XIIe-XIV ${ }^{\mathrm{e}}$ siècle, il mentionne des toponymes le long d'une rivière (probablement la Sirumambe car le toponyme Batu Gana y figure), un sima dans la région de Nan Mularang et le nom de Kudhi Haji 27.

- une statue de lion en tuf collectée à Tandihat II. L'inscription d'une ligne incisée sur la cuisse gauche de l'animal est en vieux-javanais utilisant des caractères vieux-javanais. Daté paléographiquement XIIe-XIVe siècle, ce texte est la formule «Buddha i swakarmma» 28 .

- un fragment de statue de lion ${ }^{29}$ collectée par Schnitger à Tandihet I. L'inscription comporte deux lignes en caractères vieux-javanais illisibles selon lui ${ }^{30}$. Ce texte semble effectivement n'avoir jamais été déchiffré.

Pour résumer, à ce jour ce corpus des inscriptions de Padang Lawas montre l'usage de quatre systèmes d'écriture distincts : le vieux-javanais, majoritaire (six), suivi du nāgarī (deux), du vieux-batak (deux), et enfin du tamoul (un). Les langues sont tout aussi nombreuses, puisqu'on y retrouve le vieux-malais, majoritaire (six), le sanskrit (trois), le tamoul et enfin le vieuxjavanais. À notre avis, dans tout le monde malais, une telle diversité ne se retrouve qu'à l'échelle de la province actuelle de Sumatra Ouest. Cet ensemble amène ainsi d'entrée la question des rapports de la région avec l'Asie du Sud, Java et le pays minangkabau.

25. Coll. Musée National de Jakarta, no. 6149 (Schnitger, 1936a : 14; 1937a : 22; Stutterheim, 1937 : 148-150, 159, pl. IV; Boechari, 1985/6 : 214).

26. Coll. Musée National de Jakarta, no. 6146 (Schnitger, 1936a : 1, note 1, p. 38; 1989 : 70; Anonyme, 1936 : 199; Stutterheim, $1937: 147-148,158$, pl. IV; Boechari, 1985/6 : 213). Le lien avec Yamari est contesté par Nik Hassan Shuhaimi (1992: 81). Par ailleurs, Setianingsih y lit le chronogramme 1314 S (1392 EC) (Setianingsih et al., $2003: 5-6$ ).

27. Coll. musée provincial de Medan, no. 2186 (Setianingsih, 2001 : 92; Setianingsih et al., $2003: 7)$.

28. Coll. musée provincial de Medan, no. 1943 (Setianingsih, 2003 : 8-9).

29. Très probablement en tuf.

30. Coll. Musée National de Jakarta, no. D.166 (Anonyme, 1936 : 199; Schnitger, 1937a : 19). 


\section{Datation des monuments}

Bosch est le premier à avoir suggéré une hypothèse de datation des sanctuaires. Se basant principalement sur l'importance apparente du bouddhisme tantrique dans les inscriptions et la statuaire, il fit coïncider ce moment avec les règnes de deux adeptes de ce courant, celui de Krtanagara à Singasari (1254-1292) et celui d'Ādityawarman, d'abord à Jambi puis dans les hautes terres de Sumatra Ouest, entre le milieu du XIVe siècle et 1375 environ ${ }^{31}$.

Schnitger, se fondant sur des comparaisons de statuaire et les inscriptions datées, proposa d'abord une datation plus ancienne, distinguant une première phase (fin du $\mathrm{X}^{\mathrm{e}}$ siècle-début du XIe siècle) marquée par la construction des plus grands sanctuaires (Si Pamutung, Bahal I et Sangkilon) et une seconde phase au XIII siècle $^{32}$. Il revint sur cette hypothèse quelques années plus tard en situant la grande période de construction aux XII ${ }^{\mathrm{e}}$ et XIII ${ }^{\mathrm{e}}$ siècles ${ }^{33}$.

Dans les années 1950, la question fut reprise par Bernet Kempers, qui suggéra une datation postérieure au Lokanātha de bronze et, reprenant l'idée de Bosch, précisa qu'une partie des sanctuaires furent bâtis à l'époque de Krrtanagara et de Ādityawarman ${ }^{34}$.

Damais, quant à lui, privilégia l'association de Padang Lawas avec le royaume de Panai, cité dans l'inscription de Thanjavur parmi les conquêtes du royaume cōla en 1025, ainsi que dans le Nāgara-Kĕrtāgama (1365), pour placer les constructions entre ces deux dates ${ }^{35}$.

L'hypothèse la plus récente vient de Miksic qui date les sanctuaires entre le XIe siècle et le XIII" ${ }^{\text {siècle }}{ }^{36}$. Les éléments recueillis lors des récents travaux de restauration et de dégagement dans six complexes n'ont malheureusement pas débouché sur des hypothèses de datation plus fines.

\section{Les influences}

La première grande idée sur le sujet fut émise par Krom qui suggéra que 1'architecture et la sculpture de Padang Lawas ont beaucoup plus de points communs avec des productions d'Inde du Sud et d'Asie du Sud-Est qu'avec $\mathrm{Java}^{37}$.

31. Bosch, 1930 : 147. Cette hypothèse est émise peu après le travail de Moens sur le bouddhisme à Java et à Sumatra à cette époque (Moens, 1924).

32. Schnitger, $1937 \mathrm{a}: 37$.

33. Schnitger, $1989: 66$.

34. Bernet Kempers, 1959 : 75-76.

35. Damais, 1963 : 541.

36. Miksic, $2004: 247$.

37. Krom, 1923, II : 423. 
Bosch reprit cette question des rapports avec Java quelques années plus tard, soulignant que si certains traits de l'architecture et de la sculpture de Padang Lawas rappellent les monuments de Java central (soubassement quadrangulaire avec galerie et balustrade, escaliers avec makara (photos 3 et 4), lions assis (photo 5), raksasa) et de Java est (plan asymétrique du complexe, modénature du soubassement, position des gardiens, posture debout, costume à kain panjang), le résultat s'en distingue nettement ${ }^{38}$. Cette opinion, jamais contestée par la suite, fut complétée par d'autres comparaisons. À propos de la terrasse-pendopo devant le sanctuaire principal, Suleiman ajouta que cette configuration se retrouve à l'époque de Singasari ${ }^{39}$, mais qu'elle diffère par sa forme : carrée à Padang Lawas, rectangulaire à Java Est ${ }^{40}$. Le lion, figure très présente à Padang Lawas, aussi bien dans les rares basreliefs que dans la sculpture, est également fréquent à Java Central ${ }^{41}$, en particulier à Borobudur, Prambanan, Ngawen (Muntilan) et Pendem (Grabag), mais serait inconnu dans les temples de Java Est ${ }^{42}$. Callenfels fut le premier à mentionner ce qui serait une particularité des makara de Padang Lawas, à savoir la présence d'un personnage dans la gueule ouverte ${ }^{43}$, mais Schnitger rappela, plus tard, que cette particularité existe également dans les sanctuaires de Jambi ${ }^{44}$. Quant aux gardiens, les raksasa ou dvārapāla, Bernet Kempers fit observer leur posture debout contrastant avec celle des statues de Java Est, qui ont un genou à terre ${ }^{45}$. Une autre de leurs particularités tiendrait dans la représentation des membres supérieurs : main gauche menaçante levée avec l'index pointé vers le haut, et une arme à la main droite dans les statues de Padang Lawas. La configuration inverse serait la règle dans la statuaire javanaise ${ }^{46}$.

Contrairement à Krom, qui voyait une prédominance des affinités avec l'Inde du Sud, Bosch souligna l'absence de rapprochement direct avec l'architecture des temples de cette région ${ }^{47}$. Ce fut aussi l'avis de Bernet Kempers ${ }^{48}$. Plus récemment, c'est à nouveau 1'Asie du Sud qui fut mise en

38. Krom émettra le même avis peu après (1931: 302).

39. Candi Kidal et Candi Jawi. Elle mentionne aussi Panataran. Ces trois temples sont datés

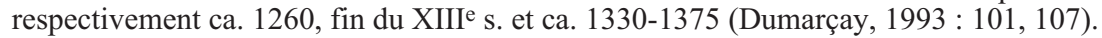

40. Suleiman, $1985: 31$.

41. Callenfels, $1920: 66$.

42. Hors de Java, des sculptures de lions existent à Bumiayu, Sumatra Sud (Satari, 2002).

43. Callenfels, 1920 : 66-67. Callenfels reviendra en 1925 afin d'inspecter les travaux de dégagement des sanctuaires de Sitopayan et de Bahal I (Callenfels, 1925; Haan, 1926).

44. Schnitger, 1937a : 17.

45. Bernet Kempers, 1959 : 76. Il s'oppose sur ce point à Bosch.

46. Mulia, $1980: 25$.

47. Bosch, 1930 : 145-147.

48. Bernet Kempers, 1959 : 76. 


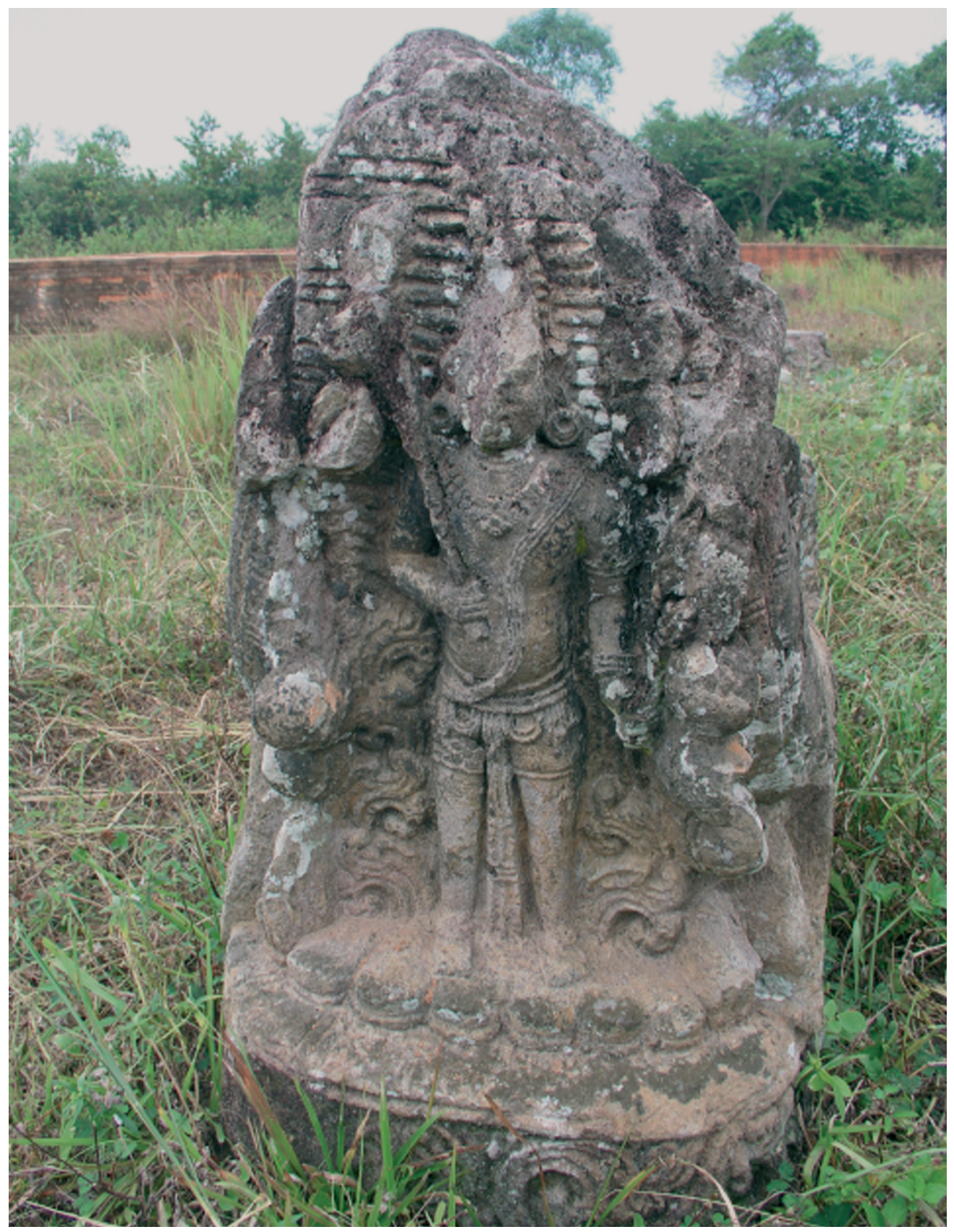

3. Si Pamutung: makara vu de face 


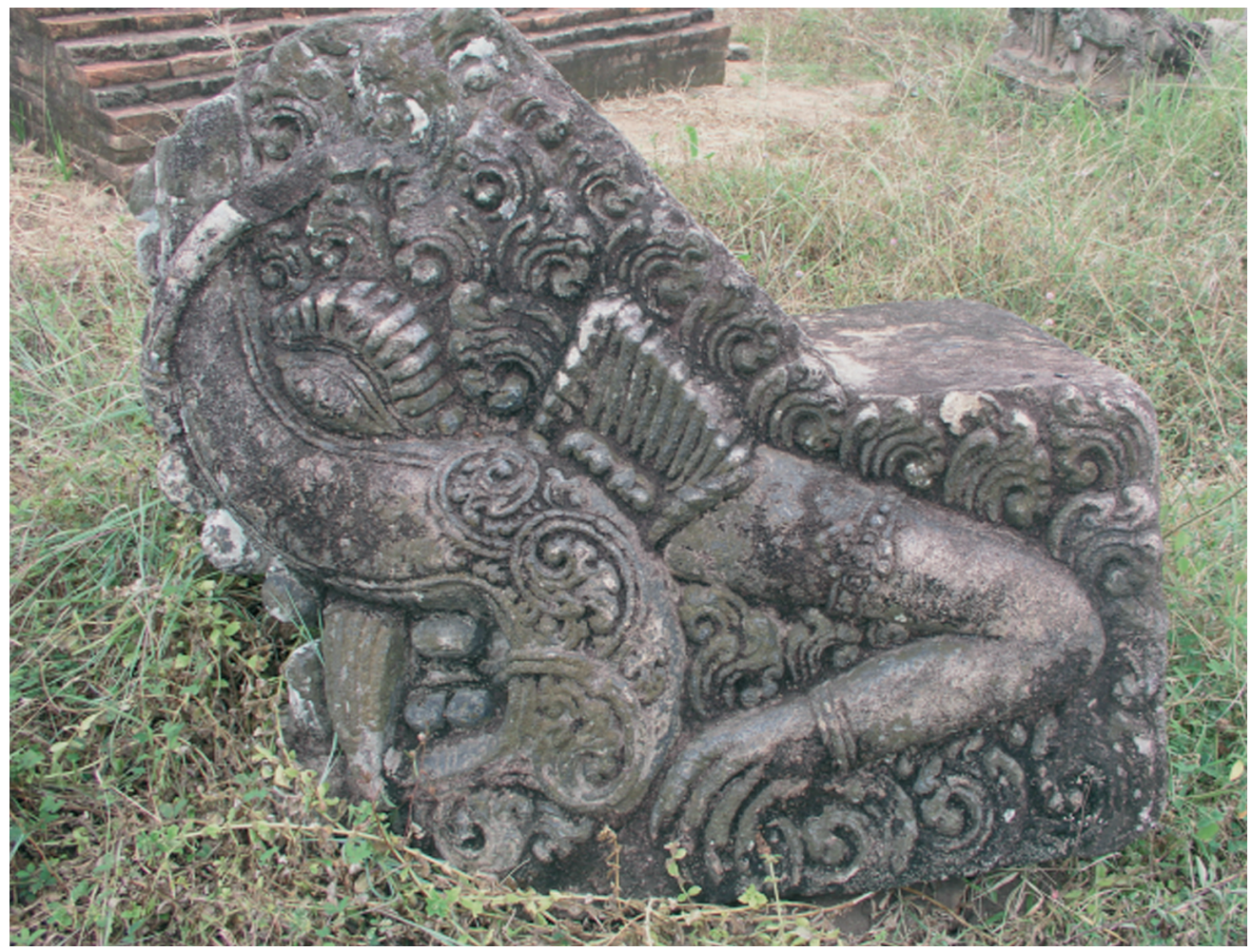

4. Si Pamutung : makara vu de côté 


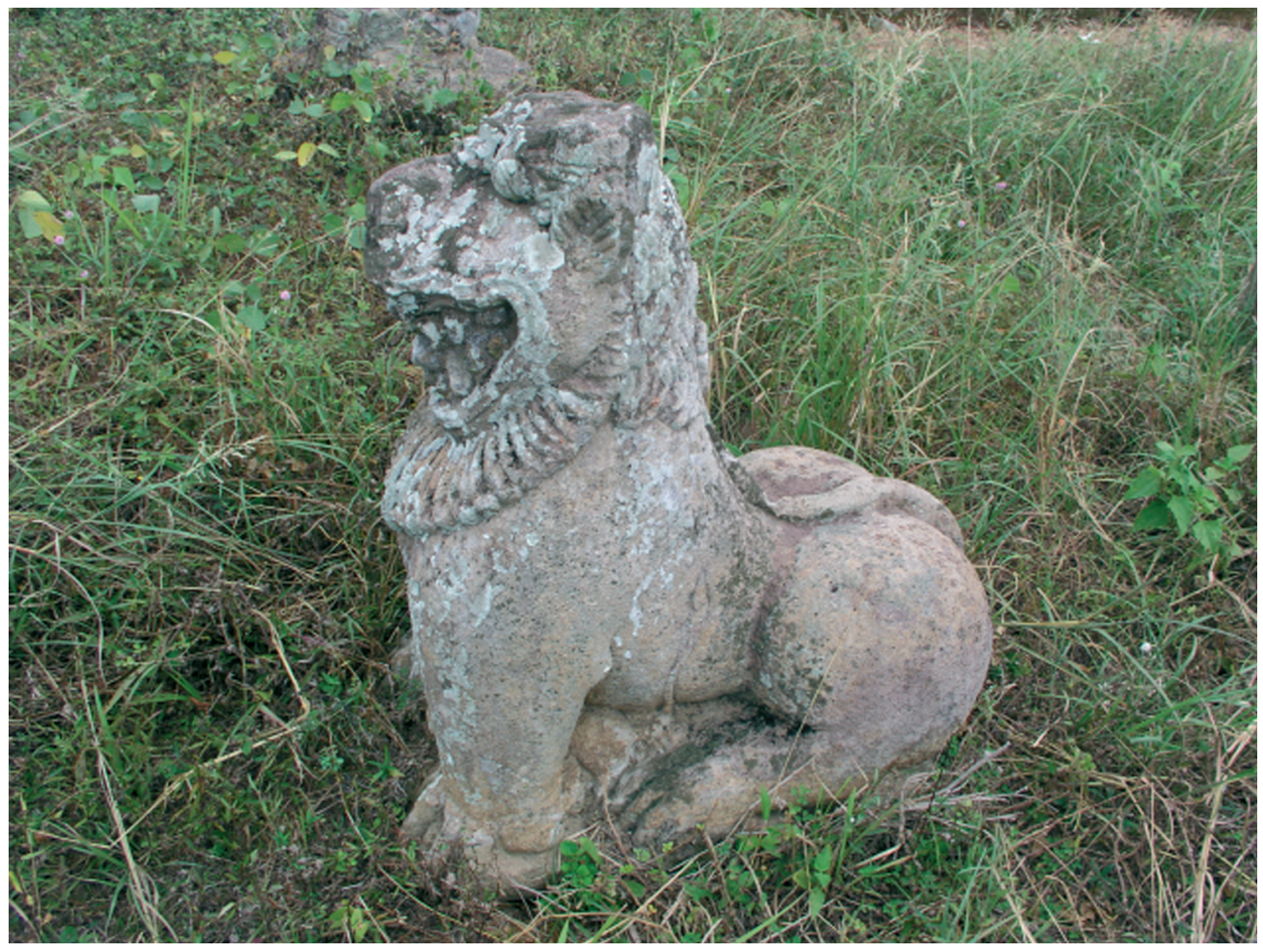

5. Si Pamutung : lion en tuf 
avant. McKinnon et Miksic parlent en effet d'une architecture fortement influencée par l'Inde, voire typique de l'Inde du Sud, sans avancer toutefois d'argument précis pour étayer cette affirmation ${ }^{49}$.

D'autres sources d'inspiration ont été évoquées à propos des sculptures de Padang Lawas : pour les lions, l'art cam et l'art de Polonnaruva à Sri Lanka ${ }^{50}$; pour les makara, également l'art cam, mais aussi l'art khmer et l'Inde du Sud ${ }^{51}$, enfin l'Inde du Sud et l'Orissa pour un gajahsimha ${ }^{52}$.

En ce qui concerne l'art du bronze, un objet en particulier a suscité des opinions diverses sur ses sources d'inspiration. Il s'agit du groupe à trois personnages avec le Lokanātha au centre, déjà mentionné dans les inscriptions, et à propos duquel les avis oscillent entre Java ${ }^{53}$ et l'art cōḷa ${ }^{54}$. Par contre, un rapprochement direct avec l'Inde du Sud fait l'unanimité pour une statuette féminine trouvée dans le complexe de Bahal I ${ }^{55}$. Deux autres pièces semblent également fortement influencées par l'Inde du Sud : un nimbe de Bara 56 et une statuette de Bouddha debout provenant de Tandihat, aujourd'hui conservée au musée de Medan ${ }^{57}$.

Dans l'état actuel des connaissances sur son architecture et sa sculpture, Padang Lawas offre donc une situation sans doute comparable à celle de la majorité des sites indianisés du monde malais et, plus généralement, d'Asie du Sud-Est. On y découvre des affinités avec diverses écoles tout en reconnaissant son originalité. Cette constatation pose le délicat et passionnant problème de l'origine, ou des origines, de ses bâtisseurs et artisans.

\section{Les religions pratiquées}

Au stade initial de notre recherche, il n'est pas question de rentrer dans le détail des débats sur les doctrines bouddhiques et hindouistes soulevées par les vestiges de Padang Lawas. Nous percevons en effet chez la plupart de ceux qui ont étudié ces vestiges une tendance à des généralisations excessives à partir de quelques indices, tendance qui brouille une réalité certainement plus complexe. Rappelons que l'image que nous avons de ces

49. McKinnon, $1984: 32$; Miksic, $2004: 247$.

50. Schnitger, 1936a : 6 ; Miksic, $2004: 247$.

51. Parkin, $1978: 84$; Mulia, $1980: 22$.

52. McKinnon, $1984: 32$.

53. Schnitger, 1937a : 32, pl. XL; McKinnon, $1984: 32$.

54. Suleiman, 1981 : 14; Nik Hassan Shuhaimi, 1992 : 76; Hardiati, 1997 : 241.

55. Coll. Musée National, Jakarta, no. 5887. Bosch, 1930 : 137, pl. 38; Sastri (1936 : 106107), qui la date du $X^{\mathrm{e}}$ siècle; McKinnon (1984:32), qui ajoute l'Orissa comme source d'inspiration possible.

56. Coll. Musée National, Jakarta, no. 6115 (Anonyme, $1936: 200$; Schnitger, 1937a : 30, pl. XXXIV; $1989: 80-81$, pl. VIII).

57. Hardiati, $1997: 242$. 
monuments aujourd'hui est loin d'être complète, non seulement en raison des destructions et des disparitions d'indices, mais également en raison des stades d'analyse très inégaux : quatre complexes sont entièrement restaurés alors que d'autres attendent encore les premiers sondages. Par ailleurs, excepté peut-être Tandihet I avec son inscription datée 1179 EC, aucun monument ne dispose d'une datation absolue et les rares tentatives de chronologie relative sont encore très hypothétiques et très partielles, alors que le plus ancien monument pourrait être séparé du plus récent par un intervalle de quatre siècles. Considérer dans ce contexte, où un sanctuaire a pu fonctionner pendant plusieurs centaines d'années, que tout le mobilier religieux du complexe auquel il appartient est un assemblage unique, a toutes les chances de conduire à des interprétations erronnées. Il faut également tenir compte du fait que, par définition, ce mobilier voyage et peut se retrouver dans un lieu par accident. Il y a enfin la question de l'éventuelle réappropriation locale des panthéons hindouistes et bouddhiques. Nous nous en tiendrons donc ici à une brève synthèse sur les données acquises et les hypothèses formulées à ce jour.

Sur le plan architectural, les auteurs ont retenu, comme indéniablement bouddhiques, la superstructure de certains sanctuaires (stūpa, motif de guirlande, niches à Bouddhas et chattra à Bahal I; deux étages à rangées de petits stūpa, ainsi que des chattra à Si Pamutung; chattra à Tandihat I et à Pulo), ainsi que la forme de certains monuments secondaires (stūpa à Bahal I; stüpa et chattra à Si Pamutung; stūpa à Tandihat I).

Parmi le mobilier, la trouvaille la plus importante de Padang Lawas à ce jour est certainement la représentation en haut-relief d'Heruka retrouvée dans la cella de Bahal II et identifiée par Bosch. C'est l'un des dieux les plus puissants du bouddhisme Vajrayāna et Kālacakra, maître de la secte tantrique des adorateurs de Bhairava réputés pour leurs rituels comportant des orgies et des sacrifices humains avec consommation de sang 58 . Schnitger data cette sculpture du $\mathrm{X}^{\mathrm{e}}$ siècle en raison de grandes similitudes avec une statue du Bihar de cette époque ${ }^{59}$. Bosch trouva un autre indice du Vajrayāna, cette fois à Bahal I, sous la forme d'une statuette en pierre du bodhisattva Vajrasattva ${ }^{60}$. Schnitger confirma l'importance du bouddhisme tantrique à Padang Lawas en découvrant des symboles de vajra dans d'autres complexes (Sangkilon, Tandihet I, Tandihet II et Pulo). À Sangkilon même,

58. $1930: 140-143$. Reconstituée à l'époque à partir de multiples fragments, cette figure a aujourd'hui disparu. Les différents sons qui apparaissent dans la plaquette d'or inscrite de Tandihet II feraient référence au rire rituel accompli durant certaines cérémonies tantriques (Stutterheim, 1937 : 148-150). Bosch trouva également dans la cella du même sanctuaire une statue de divinité féminine aux yeux exorbités portant un vajra (1930:140-143).

59. Schnitger, 1937a : 26.

60. Bosch, $1930: 136$. 
l'inscription sur plaquette en or révélerait un culte à Yamari, l'une des figures les plus démoniaques du bouddhisme ${ }^{61}$, tandis qu'à Si Pamutung, un torse féminin à tête couronnée, et présentant deux défenses sortant de la mâchoire supérieure, commémorerait une consécration royale en tant que Bhairavī ${ }^{62}$. Une autre figure trouvée dans le même complexe appartiendrait au même courant : il s'agit d'une tête à coiffure soignée en «écailles de poisson » portant un chapelet de crânes ${ }^{63}$. Un autre complexe, Bahal III, a également livré récemment un fragment de statue de Bhairava avec deux défenses ${ }^{64}$. Les cinq jina ou dhyānibuddha sont aussi présents à Padang Lawas : Amitābha à Si Pamutung et Tandihat I; Ratnasambhava (ou Aksobhya) et Amoghasiddhi à Tandihat I; Vairocana à Bahal III 65. Il est probable que la statuette de Maitreya, en céramique chinoise de la fin des Song, retrouvée à Tandihat II ${ }^{66}$, ainsi que l'ensemble en bronze inscrit représentant Avalokiteśvara-Lokanātha soient également associés à ce courant tantrique. Les stambha sont relativement nombreux à Padang Lawas ${ }^{67}$. La fonction de ces colonnes reste à éclaircir dans le contexte de Padang Lawas. Suleiman y voit des biaro miniatures, équivalents des candi miniatures de l'art de Java Est au XIVe siècle ${ }^{68}$. Celle de poteau sacrificiel n'est pas à exclure. Certains sont clairement associés au bouddhisme tantrique ${ }^{69}$. Mais le stambha n'est pas exclusivement bouddhique. Il intervient ainsi dans l'hindouisme, en tant que symbole du lien entre ciel et terre ou en tant que monument commémoratif de victoire. Notons enfin que des représentations de Bouddha ont été identifiées à Bahal II et à Tandihat ${ }^{70}$.

Padang Lawas a également livré des éléments considérés comme typiquement hindouistes. Les plus nombreux sont les représentations de Ganeśa. Le premier connu est un Ganeśa debout provenant de Portibi ${ }^{71}$, qui entra dans les collections de la Bataviaasch Genootschap en 187772. En 1920, à Porlak Dolok, Callenfels observa à la fois la représentation de Ganeśa inscrite, men-
61. Schnitger, $1989: 70$.
62. Schnitger, 1936a: 6.
63. Nik Hassan Shuhaimi, $1992: 80$.
64. Utomo, $1996: 77$.
65. Schnitger, 1936a : 4, 10; Setianingsih, $2001: 79,92,96$.
66. Schnitger, 1937a : 22 .

67. Des stambha ont été identifiés à Sitopayan, Hayuara, Bahal I, Tandihat I, Tandihat III, Nagasaribu, Mangaledang, Padangbujur, Sihodahoda et Sisoldop.

68. Suleiman, $1985: 33-34$.

69. À Tandihat I (Schnitger, 1936a : 10).

70. Bosch, 1930 : 143-144; Hardiati, $1997: 242$.

71. Sans doute originaire de l'un des complexes de Bahal, puisque Portibi en est très proche.

72. $N B G, 1877: 36$. 
tionnée plus haut, et une statue de Ganeśa à quatre bras ${ }^{73}$. Une statue inachevée de Ganeśa a été retrouvée à l'intérieur du complexe de Bahal II, lors des premiers travaux de restauration en $1982^{74}$. On lui associe un fragment de statue de Nandīíwara ou de Mahākāla ${ }^{75}$. Suleiman ${ }^{76}$ mentionne la découverte d'une statue de Ganeśa à Sangkilon, mais ne donne pas de précision sur les circonstances de cette découverte, ce qui incite à considérer cette information avec précaution. Une statue de Śiva Mahādeva avec massue, triśūla et cordon de caste en motif de pierres précieuses a été identifiée récemment à Bara ${ }^{77}$. Elle confirme le caractère sivaïte de ce complexe, après la découverte d'un piédestal en forme de nāga par Schnitger ${ }^{78}$. Il faut ajouter à ce corpus une figure «hindouiste» non identifiée qui proviendrait du sanctuaire disparu de Gunung Tua 79 .

Deux éléments semblent combiner sivaïsme et bouddhisme, d'une part, un linga sur socle en forme de double lotus observé à Tandihat I et, d'autre part, sur le sanctuaire principal de Pulo, les bas-reliefs de nature bouddhique inspirés de la théologie sivaïte, avec notamment deux figures de taureau et d'éléphant rappelant Nandin et Ganeśáa ${ }^{80}$.

Padang Lawas semble donc avoir connu au moins trois courants religieux: bouddhisme Vajrayāna, sivaïsme et syncrétisme sivaïsme-bouddhisme. Grâce à l'inscription datée du Lokanātha, on peut raisonnablement penser que des adeptes du bouddhisme Vajrayāna étaient dans la région dès les années 1030. En datant la représentation d'Heruka du $\mathrm{X}^{\mathrm{e}}$ siècle, Schnitger situerait alors son introduction au tout début de l'occupation de Padang Lawas à l'époque historique, comme nous le verrons dans le volet suivant. L'origine et les vecteurs de ce courant restent à déterminer. Si l'on retient la datation paléographique de l'inscription sur plaquette d'or de Tandihet II, le bouddhisme tantrique pourrait avoir perduré dans la région jusqu'à l'abandon des sanctuaires, apparemment dans le courant du XIVe siècle. En ce qui concerne les deux autres courants, nous nous en tiendrons pour l'instant à observer cette présence répétée de Ganeśa, qui a pu jouer un rôle particulier à Padang Lawas. Ainsi, le commanditaire du pilier inscrit à tête de Ganeśa de Porlak Dolok pourrait être un bouddhiste portant le nom de Dipankara.

73. Callenfels, $1920: 70$.

74. Utomo, $1996: 76$.

75. Susanto, $1994: 24$.

76. Suleiman, $1985: 29$.

77. Susanto, $1995: 22-23$.

78. Schnitger, 1936a : 21.

79. Ibid. : 26.

80. Schnitger, 1936a : 11, pl. X; 1937a : 30; 1937b. 


\section{Sites d'habitat et population}

Les premières recherches archéologiques consacrées à l'habitat ancien de Padang Lawas remontent à 1999. Cette nouvelle problématique a coïncidé avec la redécouverte d'un système de levées de terre et de fossés autour du complexe de Si Pamutung à l'occasion de sa restauration ${ }^{81}$. C'est probablement cette «place fortifiée» que repéra Godon dès $1855^{82}$, puis Kerchoff une trentaine d'années plus tard ${ }^{83}$. Schnitger en a livré un plan succinct, qui tombera ensuite dans l'oubli ${ }^{84}$. Elle est également mentionnée dans certaines traditions locales ${ }^{85}$. Situé sur une hauteur surplombant le confluent des rivières Barumun et Batang Pane, Si Pamutung occupe une position stratégique. D'autant plus stratégique que, d'après une tradition locale, la partie nord du site était autrefois appelée Muara Tambang, signalant une «mine d'or » ${ }^{86}$. L'espace intérieur de ce système, qui couvre une superficie proche de trente hectares, est découpé en quatre zones délimitées par ces levées de terre et fossés (voir plan). Le sanctuaire principal de Si Pamutung occupe le centre de l'un de ces secteurs. Les dix-neuf sondages réalisés à l'intérieur de cet espace par le Bureau Archéologique de Medan entre 1999 et 2003 ont tous livré un matériel plus ou moins abondant comprenant des tessons de poteries, de céramiques importées et de verre et plusieurs d'entre eux ont mis au jour des sections de murs en briques ${ }^{87}$. À ce jour, unique site de Padang Lawas à présenter une telle configuration et un tel potentiel, Si Pamutung, qui, rappelons-le, abrite également le plus grand sanctuaire de la région, a été choisi dans le cadre de la phase 2 du programme archéologique franco-indonésien consacré aux sites d'habitat ancien de Tapanuli.

Partant du plan provisoire établi par le Bureau de Medan en 1999 et d'une prospection effectuée quelques mois auparavant, la première campagne (2006) s'est d'abord orientée vers la partie de l'aménagement la plus visible aujourd'hui, qui se trouve à l'est. Une tranchée de vingt mètres ouverte perpendiculairement à l'axe du fossé a permis de confirmer l'existence d'une levée de terre de part et d'autre du fossé (photo 6). Le dénivelé maximal à cet endroit entre le sommet de la levée et la base du fossé est estimé à

\footnotetext{
81. Susilowati, 2001.

82. Godon, 1862 : 36-37.

83. Kerchoff, $1889: 492$.

84. Schnitger, 1936a : pl. XV.

85. Parlindungan, $1964: 591$.

86. Schnitger, $1937: 25$.
}

87. Susilowati, 1999; Susilowati, Wiradnyana \& Koestoro, 2000; Susilowati, Oetomo \& Sutrisna, 2003. Deux autres complexes ont également récemment fait l'objet de sondages destinés à identifier des zones d'habitat à proximité des sanctuaires : Nagasaribu (Susetyo, 2003) et Mangaledang (Susetyo, 2006). 


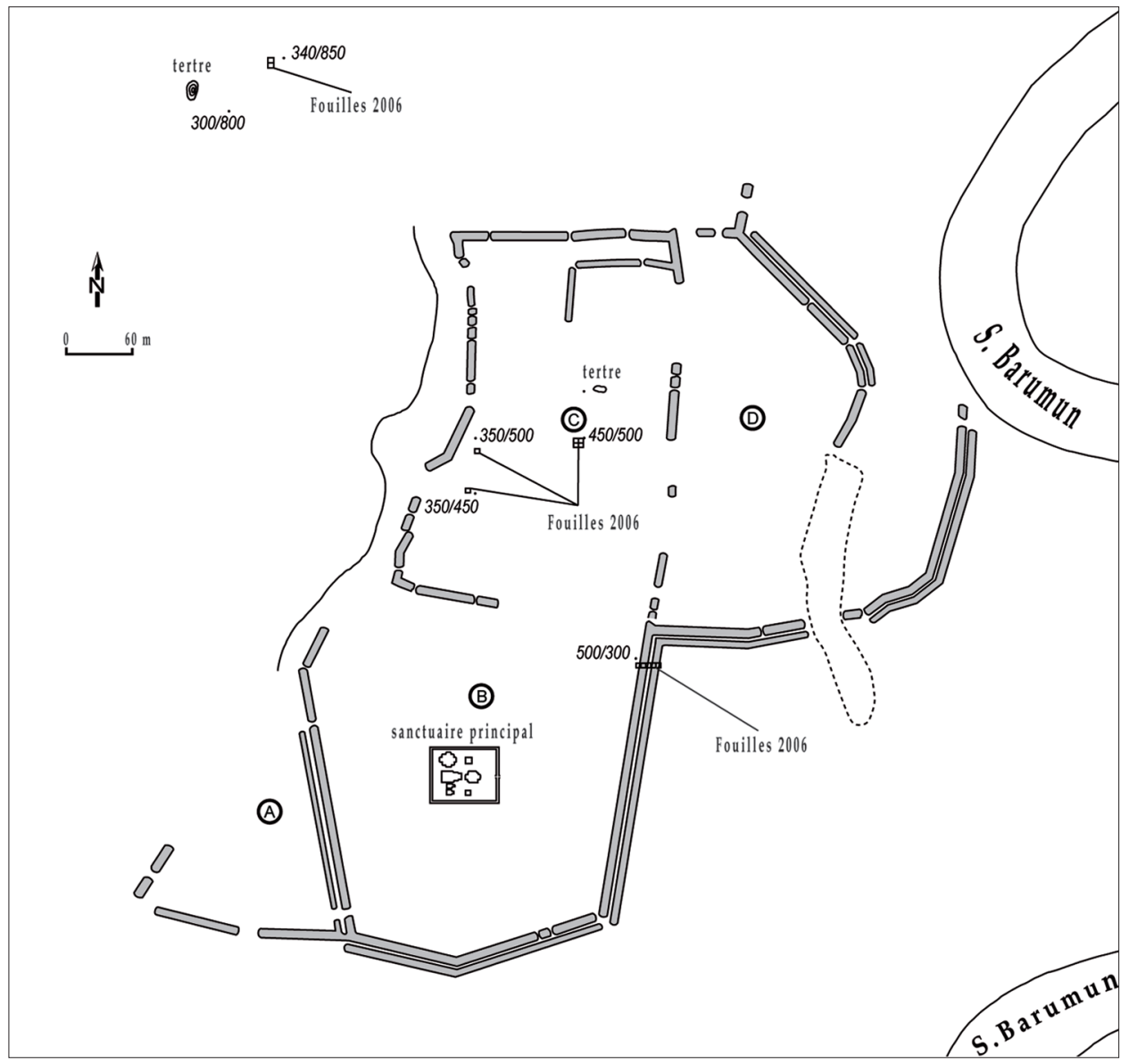

Le site de Si Pamutung (d'après Susilowati et al., 2003) 


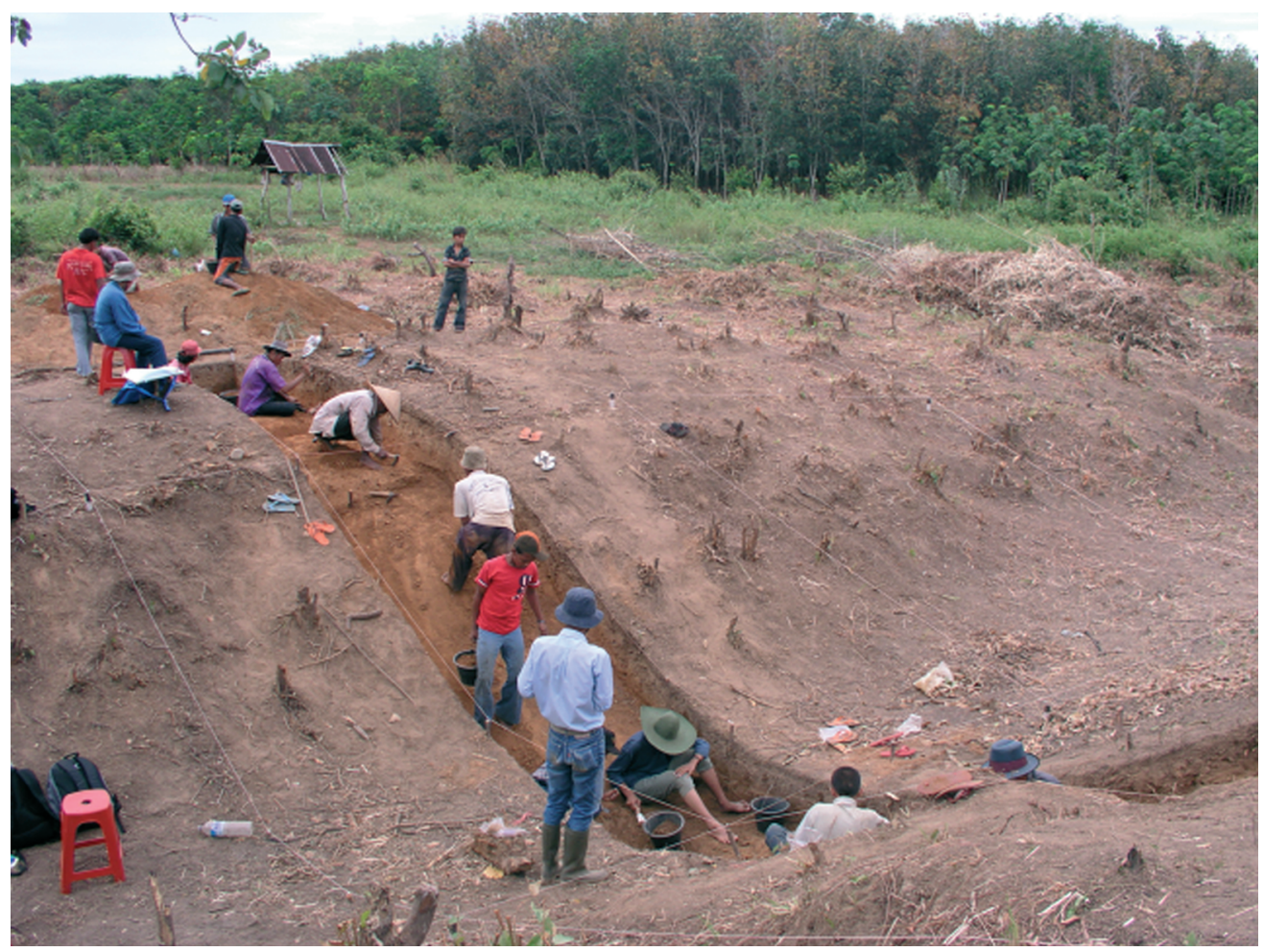

6. Si Pamutung : dégagement douve est (fouilles 2006) 
quelque quatre mètres. Ces levées de terre ne montrent pas de traces de mur en briques ou de palissade en bois. Elles étaient probablement couvertes de bambous épineux, selon une tradition attestée non seulement dans le monde malais, mais aussi dans toute l'Asie du Sud-Est. Contrairement aux sites de Lobu Tua et Bukit Hasang dans la région de Barus, la fouille de cette portion de fossé a livré extrêmement peu de mobilier, ce qui empêche pour l'instant d'émettre une hypothèse sur la datation de l'aménagement.

En s'appuyant conjointement sur les résultats des sondages réalisés entre 1999 et 2003 et sur une nouvelle prospection pédestre, on a ouvert plusieurs carrés $(5 \mathrm{~m} \times 5 \mathrm{~m})$ dans le quartier adjacent à celui du sanctuaire principal. Les deux carrés ouverts dans la partie ouest de ce quartier ont livré un mobilier relativement abondant composé de tessons de poteries et de céramiques, de fragments de verre, de quelques perles et de fragments de briques. L'occupation semble s'être effectuée ici sur un sol de cailloux à quelques 20$30 \mathrm{~cm}$ de profondeur. Cette hypothèse reste toutefois à confirmer car foyers et fosses sont absents. D'autres carrés ont été ouverts pratiquement au centre de ce quartier. La fouille a permis ici de confirmer l'existence d'un niveau d'occupation à environ $30 \mathrm{~cm}$ sous la surface actuelle. Par ailleurs, une structure carrée ( $5 \mathrm{~m} \times 5 \mathrm{~m})$ en briques a été partiellement dégagée (photo 7). Il s'agit d'un édifice extrêmement endommagé, dont il ne reste au plus que huit rangées de briques, mais les quatre murs, d'une largeur de $0,50 \mathrm{~m}$ environ, sont encore présents. S'il fait peu de doute qu'il s'agit d'un bâtiment religieux, aucun élément ne permet d'identifier sa fonction pour l'instant. Deux tessons de céramiques chinoises, appartenant à des pièces différentes, et retrouvés sous deux des murs, sont datés des $\mathrm{X}^{\mathrm{e}}-\mathrm{XI}^{\mathrm{e}}$ siècles.

$\mathrm{Au}$ nord du site, des indices laissaient présager l'existence d'une zone d'occupation hors les murs. Cette hypothèse s'est confirmée dès l'ouverture d'un premier carré, puis d'un second, qui ont livré un matériel relativement abondant fait de tessons de poteries et de céramiques, de fragments de verre et de terre cuite. Une fosse cylindrique de plus d'un mètre de profondeur a également été mise au jour. Certains fragments de terre cuite paraissent représenter une partie d'un personnage, ce qui amène à penser qu'un atelier de fabrication de statuettes a pu fonctionner à proximité. Mais cette hypothèse demande confirmation par la découverte de produits finis. Comme dans les deux carrés du quartier nord-ouest mentionnés précédemment, l'occupation semble s'être déroulée ici aussi sur un sol de cailloux, à quelques $20-30 \mathrm{~cm}$ sous la surface actuelle. Par ailleurs, les prospections ont permis de repérer deux tertres abritant une structure en briques avec des éléments architecturaux en tuf. L'un de ces tertres est situé hors les murs.

$\mathrm{Au}$ total, cette première campagne a permis de collecter notamment 32.000 tessons de poteries $(88 \mathrm{~kg}), 4.000$ tessons de céramiques importées (22 kg), 290 fragments de verre, 25 tessons de poteries glaçurées importées, 


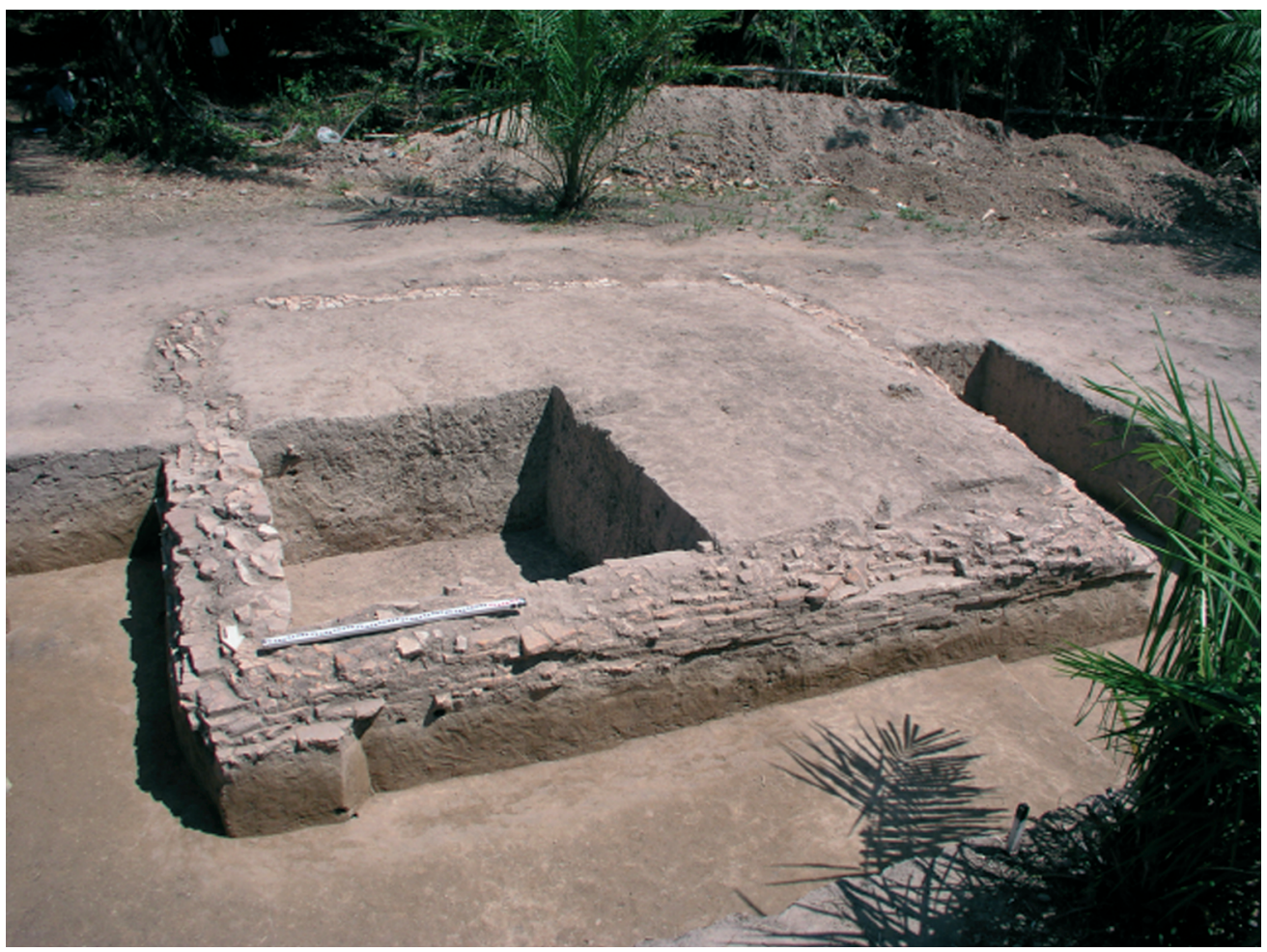

7. Si Pamutung : structure en briques dégagée lors des fouilles 2006 
24 perles et quelques 300 fragments de terre cuite (rebuts de figurines?). Il s'agit globalement d'un matériel fragmentaire montrant des traces d'usure avancée des surfaces, en particulier les céramiques. Cette observation incite à penser que le site a connu des phases d'inondation, ce qui serait une explication possible de la présence de couches de cailloux à hauteur du niveau d'occupation. Une étude géo-morphologique est prévue pour tenter d'expliquer ce phénomène et pour étudier les fluctuations des cours des rivières Pane et Barumun.

$\mathrm{Au}$ terme de cette campagne initiale, il apparaît clairement que $\mathrm{Si}$ Pamutung est un site majeur pour l'histoire de la région. Beaucoup reste à faire pour en comprendre sa structure, son organisation, ainsi que l'origine et le mode de vie de ses habitants. Les premiers jalons chronologiques de ce site d'habitat sont posés par l'analyse des céramiques ${ }^{88}$. Elle montre une occupation qui débuta dans le courant du $\mathrm{X}^{\mathrm{e}}$ siècle, prospère du $\mathrm{XI}^{\mathrm{e}}$ siècle jusqu'au début du XII e siècle, puis déclina avant de s'achever avec l'abandon du site au début du XIVe siècle. Il s'agit bien sûr d'une hypothèse provisoire, basée sur une seule catégorie d'objets, mais l'expérience de Barus a montré que les céramiques sont des marqueurs de tendance très fiables. Les sondages préliminaires et cette campagne 2006 semblent indiquer un maillage relativement dense de structures en briques. Une prospection radar est programmée pour 2007 afin d'établir un premier plan du bâti permanent et orienter les prochaines campagnes de fouilles.

La céramique montre un grande similitude avec le matériel de Barus, en particulier celui de Lobu Tua. Il en est de même pour le verre avec, dès cette première année, la collecte de fragments de verre moulés et meulés originaires du Moyen-Orient (photo 8a). Un fragment de petit flacon brun foncé opaque à filets blancs, provenant de la zone Égypte-Syrie et daté $\mathrm{X}^{\mathrm{e}}-\mathrm{XI}^{\mathrm{e}}$ siècles (photo $8 \mathrm{~b})^{89}$, figure également parmi les trouvailles. Pamutung est, à notre connaissance, le premier site de l'intérieur de Sumatra à avoir livré du verre du Moyen-Orient. S'il est clair que ce verre vient de Barus ou d'un autre comptoir sur la côte ouest, la question est de savoir qui l'utilisait. La première impression en ce qui concerne les poteries est que les assemblages sont différents à la fois de Lobu Tua et de Bukit Hasang. Les poteries d'Asie du Sud, si fréquentes à Lobu Tua, semblent nettement plus discrètes ici et la variété des formes et des décors paraît bien moindre qu'à Bukit Hasang. Une présence est notable : le kendi (gargoulette) orangé très similaire à celui probablement fabriqué à Muara Jambi ${ }^{90}$. Jambi pourrait donc avoir approvisionné Padang Lawas en poteries.

88. Cf. l'article de M.-F. Dupoizat dans ce numéro.

89. Guillot et Wibisono, $1998: 197-198$; Guillot, $2003: 259$.

90. Anonyme, $1996: 45,47$. 

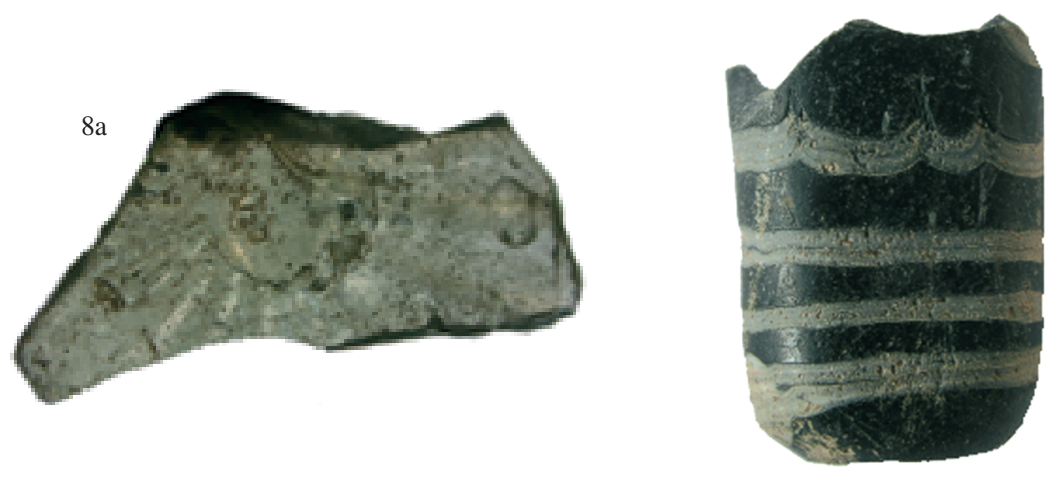

$8 b$

8a. Si Pamutung : verre soufflé moulé (Moyen-Orient) (fouilles 2006)

8b. Si Pamutung : frag. de flacon (Égypte-Syrie - Xe-XIe siècle) (fouilles 2006)

Parallèlement aux fouilles, les patronymes figurant dans les inscriptions devraient constituer une piste pour tenter de déterminer l'origine des habitants. Le terme kabayin, sur l'un des piédestals de Sitopayan, correspond certainement au vieux-javanais kabayan, signifiant «chef, dirigeant» ${ }^{91} . P u$, sang et sapta renvoient également à Java, alors que nous situerions plutôt hang et langgar dans la sphère culturelle malaise.

Par ailleurs, l'inscription en tamoul de Porlak Dolok atteste la présence de gens d'Inde du Sud à Padang Lawas au moins dès le XIIIe siècle. La toponymie de la région est également révélatrice à cet égard. Pane, Sunggam, Singkam, Pijor (et Pijar ${ }^{92}$ ) Koling renvoient tous au pays tamoul. Rappelons aussi que Bahal, toponyme signalé à deux reprises au moins à Padang Lawas, désigne le monastère bouddhique au Népal. L'histoire des marga, les groupes de filiations patrilinéaires, de Tapanuli doit également être examinée. Kern a été le premier, dès 1903 , à souligner les noms dravidiens de plusieurs subdivisions de la marga Sembiring au nord du lac Toba. Nous pensons qu'il faudrait s'intéresser de même à des marga de Tapanuli dont les noms semblent avoir des consonnances tamoules, telles que Daulay, Pane ou encore Pulungan. Par ailleurs, McKinnon a mentionné l'existence du toponyme Dhauli en Orissa ${ }^{93}$.

91. Zoetmulder, 1995, I : 434.

92. Pijar est lié au travail de forge du métal. Pijar-pijar est le borax, un sel de sodium utilisé pour ses propriétés de fondant permettant d'abaisser le point de fusion de l'or.

93. Mc Kinnon, 1984 : 32. Rappelons ici que des fragments de sculptures en gneiss à grenats, une pierre typique de l'Orissa, ont été retrouvés à Barus, lors des fouilles de Lobu Tua (Pour des exemples de sculptures anciennes de l'Orissa taillées dans cette pierre, cf. Okada, 2000 : 97-101). 
Une autre question importante se pose à propos de Si Pamutung : s'agit-il d'un centre uniquement religieux dont les habitants, permanents ou temporaires, ne seraient que des moines, des pélerins et des bâtisseurs? Ou bien s'agit-il d'un carrefour marchand, voire un centre politique, où l'espace religieux serait d'une grande importance?

Bosch est sans doute le premier à avoir fait le lien entre les vestiges de Padang Lawas, situés pour partie le long de la rivière Pane (affluent de la Barumun), le Pane de l'embouchure de la Barumun, à quelque 200 kilomètres en aval, et le Panai conquis par les Cōlas, lors de l'expédition de $1025^{94}$. Pour lui, l'origine tamoule du toponyme Panai, qui signifie «terre défrichée» ou «colonie», ne peut s'expliquer que par la présence d'une colonie tamoule installée là depuis un certain temps, probablement des marchands utilisant une route transinsulaire ${ }^{95}$. L'inscription de Thanjavur indique seulement que Panai est «arrosé par la rivière» 96 . Si ce Panai est Si Pamutung, on s'attendrait à ceque l'inscription parle non pas de «la» mais «des» rivières, puisque le site est au confluent de la Barumun et de la Pane. Dans l'état actuel des connaissances, ce seul indice inclinerait donc à penser que le Panai de l'inscription serait plutôt près de l'embouchure de la Barumun. Une prospection, effectuée en 2006, dans le cadre de ce programme Tapanuli, afin de repérer des sites d'habitat ancien entre Si Pamutung et l'embouchure actuelle s'est avérée négative. Cette question serait bien sûr à reprendre dans le cadre d'une étude sur les fluctuations du cours inférieur du fleuve. Pour l'instant donc, le lien entre l'inscription de Thanjavur et le bassin du fleuve Barumun est purement toponymique.

Toujours en rapport avec Pane, la seconde grande source mentionnée immanquablement à propos de Padang Lawas est le Nāgara-Kĕrtāgama (1365), qui cite Pane parmi les «tributaires» ou «voisins» de Mojopahit 97. Après quoi, Pane disparaîtrait, marquant aussi la fin de l'histoire de Padang Lawas durant la seconde moitié du XIVe siècle. Le texte javanais nous semble livrer cependant une autre vision de la situation à cette époque. Il mentionne notamment le toponyme «Lwas», qui pourrait bien correspondre à Padang Lawas ${ }^{98}$, même si ce toponyme est relativement fréquent à Sumatra 99 . Par ailleurs, il atteste déjà d'une certaine complexité géo-poli-

94. Auparavant, le lien était fait uniquement avec le Pane de l'embouchure de la Barumun (cf. par exemple Cœdès, 1918 : 9; Krom, 1926 : 245). Par ailleurs, des traditions locales ne relient pas Padang Lawas avec Pane mais avec Aru (Parlindungan, 1964 : 590-592, 618; Lubis, $1993: 3,31)$. Sur la question de la localisation d'Aru, cf. Perret, $1995: 70-71$.

95. Bosch, $1930: 147-148$.

96. Cœdès, $1918: 5$.

97. Pigeaud, 1960, I : 11, Canto 13 stanza 1.

98. Ibid. : Canto 13 stanza 2.

99. Il y a par exemple un Padang Lawas près de Bukittinggi. 
tique à Tapanuli puisqu'apparaît le toponyme «Mandahiling», aujourd'hui au sud de Padang Lawas, et il faut probablement lui associer aussi «Tĕba», peut-être le «Toba» actuel au nord de Padang Lawas. Ajoutons à propos de Pane que son histoire ne s'arrête pas au XIVe siècle. Il apparaît en effet comme une dépendance d'Aceh dans une lettre de 1615 adressée par Iskandar Muda au roi James I d'Angleterre (1615) ${ }^{100}$. Cette relation entre Aceh et Pane introduit la pointe nord de Sumatra dans le paysage historique de Padang Lawas. La fin de Padang Lawas serait-elle liée à l'expansion du sultanat de Pasai? Se pose également la question des rapports éventuels avec la grande zone de sanctuaires et d'habitats contemporains de Kedah en péninsule malaise, précisément dans la vallée de la Bujang ${ }^{101 .}$

Une autre piste intéressante est que Lwas, comme plusieurs autres toponymes, est décrit dans le Nāgara-Kĕrtāgama comme une dépendance de Malayu. Même si une telle vision des choses est certainement erronée pour une partie d'entre eux, elle n'est pas à exlure en ce qui concerne Padang Lawas. On sait que les premiers témoignages occidentaux sur la région au sud du lac Toba font état de traditions de soumission au puissant roi de Minangkabau ${ }^{102}$.

Il faut revenir à ce propos sur les inscriptions de Porlak Dolok (datée entre 1213 EC et 1265 EC) et de Sitopayan décrites précédemment, qui toutes deux mentionnent l'offrande à un roi portant le titre de «Pāduka Srī Mahārāja». S'agit-il d'un souverain local ou extérieur à la région? Pour la période qui nous intéresse ici, à savoir les $\mathrm{X}^{\mathrm{e}}-\mathrm{XIV} \mathrm{e}^{\mathrm{e}}$ siècles, on sait que ce titre apparaît à Java Est dans les inscriptions de Kamban (941 EC) et de Wurandungan I (948 EC), qui concernent Pu Sindok; celle de Ceker (1185 EC), qui concerne Kāmeśwara; dans plusieurs inscriptions de Krtajaya (1190-1205 EC), et enfin dans l'inscription de Tuhanaru (1323 EC), qui concerne le souverain de Mojopahit, Śrī Sundarapāṇḍadewādhīśwara ${ }^{103}$. Un autre indice nous conduit vers une connexion royale javanaise. Il s'agit de l'emblème (candra kapala) représenté sur l'inscription de Tandihat I (1179 EC). On sait qu'un souverain au moins du royaume de Janggala-

100. Gallop, à paraître.

101. Le site de Kampung Pengkalan Bujang a pu se développer à partir du $\mathrm{X}^{\mathrm{e}}$ siècle et être florissant du XIIe jusqu'à la fin du XIII ${ }^{e}$ siècle, voire jusqu'au début du siècle suivant. Les sites de Kampung Simpor Tambang et Kampung Sireh seraient contemporains de Pengkalan Bujang, avec Kampung Sireh qui aurait pris le relais de Kampung Pengkalan Bujang avant de disparaître au profit d'autres ports de la péninsule (Lamb, 1961 : 29, 83; Lamb, 1964 : 78 ; Nik Hassan \& Othman 1992 : 102, 104 ; Jacq-Hergoualc'h, 1992 : 210, 300, 303).

102. Perret, $1995: 103-104$.

103. Damais, 1952 : 61, 63, 71, 75. Plusieurs souverains de Bali ont également fait usage de ce titre entre $1098 \mathrm{EC}$ et $1200 \mathrm{EC}$, puis à la fin du XIVe siècle (ibid. : 93-95, 97). On voit mal, toutefois, comment un souverain balinais pourrait être l'objet d'offrandes à Padang Lawas. 
Kadiri (1052-1222) a utilisé ce symbole dans ses inscriptions, Bāmeśwara en 1135 EC ${ }^{104}$. Malheureusement, le souverain régnant à l'époque de l'inscription de Tandihat I, n'est pas connu avec certitude. Ce ne peut être Kāmeśwara, car il règna au plus tôt à la fin de l'année $1181^{105}$. Par ailleurs, les souverains connus juste avant et juste après cette date de $1179 \mathrm{EC}$ ne portant pas le titre de "Pāduka Śrī Mahārāja» mais celui de "Śrī Mahārāja», nous pensons que le souverain désigné à Tandihat, s'il est javanais, porte également la titulature de «Śrī Mahārāja». À ce stade, on peut donc dire que le souverain probablement mentionné dans l'inscription de Tandihat I est différent de celui mentionné à Sitopayan et à Porlak Dolok. Il pourrait donc y avoir au moins deux souverains javanais en relation avec les sanctuaires de Padang Lawas. Une inscription de Java nous conduit à suggérer que ce souverain se signalant par le candra kapala pourrait être Kroñcāryyadipa Gandra. Il s'agit de l'inscription de Jaring (Gurit) datée 1181 EC (1103 Śaka), toujours in situ ${ }^{106}$. À la suite du texte concernant l'inauguration d'un village franc (desa perdikan), il est fait mention d'une flotte de guerre et d'un amiral dans le royaume de Kadiri ${ }^{107}$. Une expédition maritime contre Sumatra deux ans auparavant est donc tout à fait envisageable. Par ailleurs, l'inscription de Jaring porte un emblème malheureusement déjà indéchiffrable à l'époque de Brandes.

Ajoutons pour terminer sur ces liens qui semblent avoir été étroits avec Java que, d'après son titre et son nom, le commanditaire de l'inscription de Porlak Dolok était sans doute javanais. Le grand intérêt de cette inscription est aussi son bilinguisme vieux-malais-tamoul. Cette inscription serait-elle liée à une expédition militaire conjointe partant vers le sud (Malayu?) ou en revenant? L'emploi du vieux-malais par un Javanais s'expliquerait ici du fait qu'il s'agit probablement de la lingua franca de Padang Lawas, comme le suggère la fréquence de cette langue dans le corpus des inscriptions connues à ce jour. Ce serait également une preuve supplémentaire de l'existence et de la perennité d'un vaste réseau de coopération javano-tamoul déjà suggéré à Barus pour une période antérieure 108 .

La question de l'environnement contemporain se doit d'être également abordé. En effet, l'environnement naturel particulièrement défavorable de la région est régulièrement invoqué pour suggérer que Padang Lawas n'aurait

104. Suhadi \& Kartakusuma, $1996: 47,60-61,72$.

105. Damais, $1952: 69$.

106. Dusun Jaring, Desa Kembang Arum, Kec. Surojayan, Kab. Blitar, Java Est (observation de D. Perret, septembre 2003).

107. Krom, 1931 : 297. Pour le texte complet de cette inscription, cf. Brandes, 1913 : no. LXXI; Suhadi \& Kartakusuma, $1996: 20-24$.

108. Guillot, $2003: 49,68$. 
jamais abrité une population nombreuse. Par ailleurs, on a parfois attribué cet aspect du paysage à la déforestation nécessaire à la cuisson des briques pour la construction des sanctuaires. Or, même en se basant sur une estimation forte selon laquelle, d'une part, les dix-sept complexes construits en briques abriteraient chacun l'équivalent de huit édifices à cella (le mur d'enceinte étant considéré comme équivalent à quatre édifices), d'autre part, une période de construction d'un siècle pour l'ensemble, on arrive à une moyenne annuelle légèrement inférieure à 1,5 monuments. Compte tenu de la superficie de Padang Lawas, l'impact sur l'environnement apparaît par conséquent comme très négligeable. Une étude palynologique serait utile pour livrer une image de l'environnement avant, pendant et après cette période construction.

\section{Voies de communication}

Chacun des volets abordés ci-dessus atteste que Padang Lawas n'était pas une région isolée, mais communiquait certainement avec la côte ouest et le sud. La côte occidentale recevait à l'époque des produits venant de toute l'Asie, du Proche-Orient à la Chine en passant par l'Asie du Sud, fournissant en échange des produits forestiers et de l'or disponibles en abondance dans l'arrière-pays, ainsi que probablement des produits originaires d'autres régions de l'archipel, voire de la Chine. Le sud était justement un grand fournisseur d'or. C'est également au sud que se trouvait l'important royaume de Malayu. La question reste entière pour le détroit de Malacca, sachant tout de même que le fleuve Barumun était autrefois navigable jusqu'aux sites de Padang Lawas. Pour l'instant, les seuls indices en aval de Si Pamutung se limitent à un vestige de sanctuaire en briques tout près de Binanga (Aektunjang) et à deux toponymes liés à l'Asie du Sud, Pijor Koling et Singkam, près de Langgapayung. Dans le cas où un relais marchand était bien installé à l'embouchure de la Barumun, Padang Lawas était certainement en contact terrestre avec les montagnes du nord-ouest riches en camphre et en benjoin, que l'on devait pouvoir rejoindre en remontant la rivière Pane, affluent de la Barumun. L'existence de cette voie vers le nordouest pourrait alors expliquer la présence de vestiges le long de son cours inférieur.

Nous suggérons de voir la côte ouest de Tapanuli comme une suite de relais, de Barus au nord à Natal au sud, en contact direct ou indirect avec Padang Lawas. L'importance de Barus, entre le milieu du IX ${ }^{\mathrm{e}}$ siècle et la fin $\mathrm{du} \mathrm{XI}^{\mathrm{e}}$ siècle, a été récemment démontrée. On sait maintenant, qu'après une rupture probable de quelques décennies, la ville retrouva une prospérité qu'elle conserva jusqu'au début du XVe siècle. Si l'on s'en tient aux sources étrangères contemporaines, Barus était certainement la place marchande la plus importante de la côte ouest de Tapanuli à l'époque de Padang Lawas. 
C'est surtout la présence de verre du Moyen-Orient à Si Pamutung qui, à ce stade, nous amène à penser à un lien direct, possible mais compliqué, ou surtout indirect avec Padang Lawas, ceci par l'intermédiaire d'un port secondaire. Sorkam, à environ vingt kilomètres au sud-est de Barus, a pu également jouer un rôle. Les indices sont pour l'instant l'origine tamoule du toponyme, la découverte d'une tête de statue en pierre à la fin du XIXe siècle ${ }^{109}$, ainsi que l'existence de filons d'or dans les montagnes à la source de la rivière Aek Si Bundang, qui se jette dans l'océan Indien à Sorkam ${ }^{110}$. Là encore, même si l'existence d'une voie terrestre pour atteindre Padang Lawas est envisageable, le passage par un relais côtier situé plus au sud nous apparaît plus plausible. Notre troisième point est le golfe de Sibolga, à quelque quarante kilomètres au sud de Sorkam, en particulier l'embouchure de la rivière Lumut, où une statue de Ganeśa a été retrouvée il y a quelques années 111. L'embouchure d'une des branches du delta de la Batang Toru, à environ 60 kilomètres du point précédent serait sans doute intéressante à prospecter dans la mesure où le toponyme Batu Mundam présente une composante d'origine tamoule. De même, à l'embouchure de la Batang Gadis, à environ 20 kilomètres au sud de Batu Mundam, un endroit nommé «Picar Koling», près de Singkuang, aurait livré des "antiquités» à la fin du XIXe siècle ${ }^{112}$. Natal, enfin, le petit port à quelque 60 kilomètres au sud de Singkuang, est bien connu pour la richesse en or du bassin de la Batang Natal. La découverte d'ornements et de bagues en or par des orpailleurs travaillant dans cette rivière a été signalée ${ }^{113}$. De plus, la source de cette rivière est située au pied du volcan Sorik Merapi, au sommet duquel on a retrouvé quatre piliers inscrits en vieux-malais, dont l'un daté 1242 EC, ainsi que trois structures en briques contenant des urnes funéraires ${ }^{114}$. Selon nous, tous les points situés entre le golfe de Sibolga et Natal ont pu avoir un lien direct avec Padang Lawas, par l'intermédiaire des bassins des rivières Batang Toru, Batang Gadis et Batang Natal.

Les données sont a priori plus riches pour les liaisons avec le sud qui semblent avoir été nombreuses. On sait ainsi qu'au milieu du XIX ${ }^{\mathrm{e}}$ siècle, Portibi, près de Bahal, était accessible en deux jours à partir de Kota Nopan, en suivant les rivières Aek Mango, Siapas, Sirumambe et Batang Pane ${ }^{115}$. Ce trajet est très intéressant car il montre que la rivière Sirumambe, riche en

109. $N B G, 37,1899: 12$

110. Anonyme, $1917: 200$.

111. Koestoro, 2001.

112. $N B G, 25,1887: 56$.

113. Callenfels, $1920: 65$.

114. $N B G, 26,1888: 89,151,191 ; N B G, 31,1893: 4$; Damais, $1952: 100-101 ; 1955: 209$.

115. Godon, $1862: 56$. 
sanctuaires, a dû être empruntée de longue date en venant du sud. D'autres trajets nous paraissent balisés par des sanctuaires. Ainsi, la voie pour atteindre le bassin de la rivière Rokan devait suivre la rivière Barumun en amont de Si Pamutung, passant ainsi par le sanctuaire de Sangkilon, puis celui de Manggis, près de la source de la rivière Rokan Kanan ${ }^{116}$. Une autre voie devait bifurquer après Sangkilon pour suivre la Barumun presque jusqu'à sa source en passant par les sanctuaires de Porlak Dolok et Pageranbira. De là, un passage dans la montagne devait aboutir aux sanctuaires de Simangambat et Bonan Dolok, certainement un carrefour important, puisque de cet endroit on avait sans doute accès aux trois bassins de la côte ouest, ainsi qu'à la voie rejoignant le sud par Kota Nopan ${ }^{117}$. Selon nous, cette position privilégiée explique certainement le fait que le sanctuaire (sivaïte) de Simangambat soit le plus ancien de Tapanuli (VIIIe-IXe siècle) ${ }^{118}$.

\section{Conclusion}

Ce tour d'horizon des données et des hypothèses au démarrage du programme sur les sites d'habitat de Padang Lawas dessine les futures directions de recherche, qui sont nombreuses. Nous disposons d'un premier ancrage avec le site de Si Pamutung, probablement le site majeur de la région, stratégiquement placé au confluent de deux rivières, la Barumun et la Batang Pane, qui permettent l'accès aux richesses économiques de la chaîne des Barisan et aux produits importés disponibles sur la côte ouest et probablement sur la côte est. Mais pourquoi un tel choix d'implantation, si loin de l'embouchure de la Barumun dans le détroit de Malacca?

Avec son système complexe de levées de terre et de fossés combiné à la densité apparente de son bâti permanent, Si Pamutung a le potentiel pour livrer un plan même sommaire de son urbanisme, ce qui serait une première pour Sumatra Nord, voire à l'échelle de Sumatra. Les premiers jalons chronologiques étant posés grâce à l'étude des céramiques $\left(\mathrm{X}^{\mathrm{e}}\right.$-début $\mathrm{XIV}{ }^{\mathrm{e}}$ siècle), nous tenterons d'éclaircir notamment l'articulation des trois moments clés de son histoire : son occupation, l'aménagement du système de levées de terre et la construction du complexe religieux principal.

116. Cette section faisait certainement partie de l'ancienne grande voie mentionnée par Rosenberg au milieu du XIXe siècle, qui reliait Portibi et Dalu-Dalu sur la rivière Sosa, un affluent de la Rokan (1855: 58).

117. Plusieurs sites entre Bonan Dolok et Kota Nopan ont livré des objets anciens : statuette en bronze d'éléphant monté par deux personnages à Penyabungan (Callenfels, 1920 : 65; Schnitger, 1937a : 14); statuette en bronze de Kubera et d'un éléphant à Tano Bato, $20 \mathrm{~km}$ au sud de Penyabungan (Schnitger, 1937a : 14); lampe en bronze en forme d'oiseau de Simpang Tolang, près de Kota Nopan $(N B G, 1891: 41$, lxxxii).

118. Bosch, 1930 : 134, pl. 29a; Schnitger, 1936b; 1937a : 14. 
Le corpus des inscriptions, les influences lisibles dans l'architecture et l'art, la toponymie de Padang Lawas mettent en lumière trois composantes majeures dans la population de l'époque : sumatranaise, javanaise et tamoule. Comment ces trois composantes s'organisent-elles dans le cadre du site de Si Pamutung? Le matériel archéologique pose quant à lui la question d'une éventuelle présence chinoise, voire de gens originaires du MoyenOrient. Ces hypothèses sur le cosmopolitisme sont évidemment liées à la nature de Si Pamutung? Qui est à l'origine de son implantation? Ville monastique ou carrefour marchand? La ville dispose-t-elle d'un pouvoir indépendant ou bien fait-elle partie d'un grand ensemble politique? De ce point de vue, nous avons suggéré de fortes connexions avec au moins deux rois de Janggala-Kadiri et Malayu pourrait aussi avoir joué un rôle.

Possédant le plus grand et peut-être le plus ancien sanctuaire de Padang Lawas, Si Pamutung offre également la possibilité d'éclairer l'histoire religieuse de la région, en particulier l'articulation des trois courants décelés dans l'architecture et l'art : bouddhisme Vajrayāna, sivaïsme et syncrétisme sivaïsme-bouddhisme. Rappelons ici qu'aucun sanctuaire en briques n'a été mis au jour sur les sites de Barus contemporains de Si Pamutung. D'où vient le courant Vajrayāna? Comment s'articule cette histoire avec celle des sanctuaires de Muara Jambi, de Muara Takus et du pays minangkabau dans la moitié sud de Sumatra, celle des sanctuaires de la vallée de la Bujang en péninsule malaise? Par ailleurs, comment s'est comporté cet avant-poste hindo-bouddhique sumatranais face à l'émergence du sultanat de Pasai? La fin de Padang Lawas a-t-elle un lien direct avec ce nouveau contexte politico-religieux?

Plus généralement se posent toutes les questions des relations entre $\mathrm{Si}$ Pamutung et les autres sanctuaires de Padang Lawas, dont certains sont peutêtre aussi des marqueurs d'un habitat significatif. Sont-ils tous contemporains ou correspondent-ils à plusieurs phases d'implantation? Comment se présente l'environnement avant, pendant et après cette ou ces phases de construction? Au-delà, le suivi des voies de communication anciennes à travers le repérage des vestiges archéologiques et des indices toponymiques ouvre sur l'histoire des sites côtiers occidentaux au sud de Barus. La réunion de quelques indices nous a permis de suggérer cinq sites potentiels. Le grand comptoir encore élusif de la côte est, peut-être d'une taille équivalente à Kota Cina et probablement situé près de l'embouchure de la Barumun, fait aussi partie des chaînons manquants. Son repérage nécessitera probablement une étude géo-morphologique des fluctuations du cours du fleuve.

On voit, à travers toutes ces questions, que Padang Lawas constitue l'une des clés majeures pour comprendre l'histoire ancienne de Sumatra. 


$\begin{array}{ll}\text { Abréviations } & \\ \text { BEFEO } & \text { Bulletin de l'École française d'Extrême-Orient } \\ \text { BPA } & \text { Berita Penelitian Arkeologi } \\ \text { BRCAI } & \text { Bulletin of the Research Centre of Archaeology of Indonesia } \\ \text { FMJ } & \text { Federation Museums Journal } \\ \text { JBGKW } & \text { Jaarboek van het Bataviaasch Genootschap van Kunsten en } \\ & \text { Wetenschappen } \\ \text { JGIS } & \text { Journal of the Greater India Society } \\ \text { JMBRAS } & \text { Journal of the Malayan Branch, Royal Asiatic Society } \\ \text { NBG } & \text { Notulen van de Algemeene en Directievergaderingen van het } \\ & \text { Bataviaasch Genootschap van Kunsten en Wetenschappen } \\ \text { OV } & \text { Oudheidkundig Verslag uitgegeven door het Bataviaasch } \\ \text { TBB } & \text { Genootschap van Kunsten en Wetenschappen } \\ \text { TBG } & \text { Tijdschrift voor het Binnenlandsch Bestuur } \\ \text { TNI } & \text { Tijdschrift voor indische Taal-, Land- en Volkenkunde } \\ \text { VBG } & \text { Tijdschrift voor Nederlandsch-Indië }\end{array}$

\section{BibLIOGRAPHIE}

Anonyme, «Verslag van een reis van den controleur van Baros naar de beoosten Baros gelegen onafhankelijke landschappen in het jaar 1883», TBB, 52, $1917:$ 195-205, 252-265.

Anonyme, «De Archaeologische Verzameling», JBGKW, III, 1936 : 194-202.

Anonyme, «Berita Temuan», Amerta, 17, $1996: 42-51$.

Bernet-Kempers, A.J., Ancient Indonesian Art, Amsterdam, C.P.J. van der Peet, 1959.

Boechari, Prasasti Koleksi Museum Nasional. Jilid I, Jakarta, Museum Nasional, 1985/86.

Bosch, F.D.K., «Verslag van een reis door Sumatra», OV, 1930 : 133-157.

Brandes, J.L.A., Oud-Javaansche oorkonden, nagelaten transcripties van wijlen Dr. J.L.A. Brandes (uitgegeven door N.J. Krom), VBG, LX, 1913.

Callenfels, P.V. van Stein, «Rapport over een dienstreis door een deel van Sumatra», OV, 2, $1920: 62-75$.

- «Verslag van Dr. P. V. van Stein Callenfels over zijn inspectiereis door Sumatra», $O V$, 1/2, 1925: 11-14.

Cœdès, George, «Le Royaume de Çrivijaya», BEFEO, 18(6), 1918 : 1-36.

Damais, Louis-Charles, «Études d'épigraphie indonésienne. - III. Liste des principales inscriptions datées de l'Indonésie», BEFEO, 46(1), 1952:1-105.

-, «Études d'épigraphie indonésienne. - IV. Discussion de la date des inscriptions », BEFEO, 47(1), 1955 : 7-290.

-, «Bibliographie indonésienne. V. Publications du Service Archéologique de l'Indonésie», BEFEO, 51(2), 1963 : 535-594. 
Departemen Pendidikan dan Kebudayaan, Hasil Pemugaran dan Temuan Benda Cagar Budaya PJP I, Jakarta, Departemen Pendidikan dan Kebudayaan, 1996.

Dumarçay, Jacques, Histoire de l'architecture de Java, Paris, École française d'ExtrêmeOrient, Mémoires Archéologiques XIX, 1993.

Gallop, Annabel Teh, "Gold, silver and lapis lazuli : Royal Letters from Aceh in the 17th century», communication à First International Conference on Aceh and Indian Ocean Studies, Banda Aceh, 24-26 février 2007.

Godon, A.P., «De assistent-residentie Mandaheling en Ankola, op Sumatra's Westkust, van 1847 tot $1857 », T N I, 24(1), 1862: 1-41$.

Guillot, Claude \& Wibisono, Sonny, «Le verre à Lobu Tua. Étude préliminaire», in C. Guillot (éd.), Histoire de Barus, Sumatra, Le site de Lobu Tua. I. Etudes et Documents, Paris, Cahier d'Archipel 30 : 189-206.

Guillot, Claude (éd.), Histoire de Barus. Le Site de Lobu Tua : I. Études et Documents, Paris : Cahiers d'Archipel 30, 1998. Traduit en indonésien par Daniel Perret, Lobu Tua-Sejarah Awal Barus, Jakarta, École française d'Extrême-Orient/Yayasan Obor Indonesia, 2002.

Guillot, C. ; Surachman H.; Perret D., et al., Histoire de Barus, Sumatra, Le site de Lobu Tua. II : Étude archéologique et Documents, Paris, Cahier d'Archipel 30, 2003.

Haan, B. de, «Verslag van den Bouwkundig Opzichter», OV, 1/2, $1926: 24-28$.

Hardiati, Endang Sri, «Arca-arca Buddha, Temuan Baru dari Sumatera Utara», in I.A.M. Romli (ed.), Cinandi, Yogyakarta, Universitas Gajah Mada, 1997 : 240-243.

Jacq-Hergoualc'h, Michel, La civilisation des ports-entrepôts du sud Kedah (Ve-XIVe siècle), Paris, L'Harmattan, 1992.

Karashima, N., «Tamil Inscriptions in Southeast Asia and China», in N. Karashima (éd.), Ancient and Medieval Commercial Activities in the Indian Ocean : Testimony of Inscriptions and Ceramics-sherds, Tokyo, Taisho University, 2002 : 10-18.

Kerchoff, Ch. E.P. van, «Aanteekeningen betreffende eenige der in de afdeeling PadangLawas voorkomende Hindoe-oudheden», TBG, 32, 1889: 487-497.

Kern, H., Verspreide Geschriften. Zevende deel, 's-Gravenhage, M. Nijhoff, 1917.

Koestoro, Lucas P., «Ganesa dan Perempuan Penunggang Kuda, Dua Objek Ikonografi di Tapanuli Tengah», Berkala Arkeologi "Sangkakala" (Medan), 9, 2001 : 50-59 (Trad. part. dans Archipel, 63, $2002: 15-16)$.

Krom, N.J., Inleiding tot de Hindoe-Javaansch Kunst, 's-Gravenhage, Martinus Nijhoff, 1923, 3 vol.

—, Hindoe-Javaansche Geschiedenis, 's-Gravenhage, Martinus Nijhoff, 1931 (1 ère éd. 1926).

Lamb, Alastair, «Miscellaneous Papers on Early Hindu and Buddhist Settlement in Northern Malaya and Southern Thailand», FMJ, 6(12), 1961: 1-90.

—, «Notes on Satingphra», JMBRAS, 37(1), $1964: 74-87$.

Lubis, Mhd. Arbain, Sejarah Marga-Marga Asli di Tanah Mandailing, [Medan, 1993].

McKinnon, E.E., Kota Cina : Its Context and Meaning in the Trade of Southeast Asia in the Twelfth to Fourteenth Centuries, PhD Thesis, New York, Cornell University, 1984.

- «Historic Period Earthenware from the Island of Sumatra», in J.N. Miksic (éd.), Earthenware in Southeast Asia, Singapore, Singapore University Press, 2003 : 162-172.

Miksic, John, «The Classical Cultures of Indonesia», in I. Glover \& P. Bellwood (éd.), Southeast Asia. From Prehistory to History, London/New York, RoutledgeCurzon, $2004: 234-313$.

Moens, J.L., «Het Buddhisme of Java en Sumatra in zijn laatste bloeiperiode», $T B G, 44$, 1924 : 521-579.

Mulia, Rumbi, «The ancient kingdom of Panai and the ruins of Padang Lawas (North Sumatra)», BRCAI, 14, $1980: 1-36$. 
Nik Hassan Shuhaimi Nik Abd. Rahman, Arkeologi, Seni dan Kerajaan Kuno Sumatera Sebelum Abad ke-14, Bangi, Ikatan Ahli Arkeologi Malaysia, 1992.

-, \& Othman Mohd. Yatim, Warisan Lembah Bujang, Bangi, Ikatan Ahli Arkeologi Malaysia, 1992.

Okada, Amina, Sculptures indiennes du musée Guimet, Paris, Réunion des Musées Nationaux, 2000.

Parkin, Harry, Batak Fruit of Hindu Thought, Madras, The Christian Literature Society, 1978.

Parlindungan, Mangaradja Onggang, Tuanku Rao, [Jakarta], Tandjung Pengharapan, [1964].

Perret, Daniel; La formation d'un paysage ethnique : Batak et Malais de Sumatra Nord-Est, Paris, École française d'Extrême-Orient, 1995.

—, \& Surachman, Heddy (éd.), Histoire de Barus, Sumatra. De Pintu Ria à l'arrivée de la VOC, Paris, Cahiers d'Archipel, à paraître en 2008.

Pigeaud, Th. G. Th., Java in the 14th Century. A Study in Cultural History. The NāgaraKĕrtāgama by Rakawi Prapañca of Majapahit, 1365 A.D., The Hague, Martinus Nijhoff, 1960-1963, 5 vol.

Rosenberg, H. von, «Hindoe-Bouwvallen in het landschap Padang Lawas (Binnenlanden van Sumatra)», TBG, III, $1855: 58-62$.

Sastri, K.A. Nilakanta; «A South Indian Portrait Bronze from Sumatra», JGIS, III, 1936 : 104-107.

Satari, Sri Soejatmi, «Sebuah Situs Hindu di Sumatra Selatan : Temuan Kelompok Candi dan Arca di Bumiayu», in 25 Tahun Kerjasama Pusat Penelitian Arkeologi dan École française d'Extrême-Orient, Jakarta, École française d'Extrême-Orient \& Pusat Penelitian Arkeologi, $2002: 113-132$.

Schnitger, F.M., Oudheidkundige vondsten in Padang Lawas, Leiden, E.J. Brill, 1936a.

—, «De Tempel van Simangambat (Zuid Tapanoeli)», TBG, 76, 1936b : 334-336.

-, The Archaeology of Hindoo Sumatra, Leiden, E.J. Brill, 1937a.

—, «Five Reliefs at Poelo in Tapanoeli (Sumatra)», JGIS, 1937b : 43-44.

-, Forgotten Kingdoms in Sumatra. Introduction by John N. Miksic, Singapore, Oxford University Press, 1989 (1 1 ère ed. 1939).

Sedyawati, Edi, Pengarcaan Ganeśa Masa Kadiri dan Sịhasāri, Jakarta, LIPI-RUL, École française d'Extrême-Orient, 1994.

Setianingsih, R.M., «Vairocana dan Amitabha, Emanasi Buddha di Padang Lawas, Sumatera Utara», Berkala Arkeologi "Sangkhakala” (Medan), 9, 2001 : 91-100.

-, et al., Prasasti dan Bentuk Pertulisan Lain di Wilayah Kerja Balai Arkeologi Medan, Medan, Balai Arkeologi, 2003.

Stutterheim, W.F., «De Archaeologische Verzameling», JBGKW, IV, 1937 : 146-156.

Suhadi, Machi \& Kartakusuma, R., «Laporan Penelitian Epigrafi di Wilayah Provinsi Jawa Timur», BPA (Jakarta), 47, $1996: 1-75$.

Suleiman, Satyawati, Sculptures of Ancient Sumatra, Jakarta, Pusat Penelitian Arkeologi Nasional, 1981

- «Laporan Survei Peninggalan-Peninggalan di Sumatra Utara dalam Rangka Penyusunan Masterplan», BPA (Jakarta), 1983 : 1-52 (1 ère éd. 1976).

—, «Peninggalan-peninggalan purbakala di Padang Lawas», Amerta, 2, 1985 : 3-37 (1 1 ère éd. 1954).

Susanto, R.M. et al., Laporan Hasil Penelitian Arkeologi Situs Padang Lawas, Kabupaten Tapanuli Selatan, Medan, Balai Arkeologi, 1994.

-, Laporan Hasil Penelitian Arkeologi Ekskavasi Candi Bara, Padang Lawas, Kabupaten Tapanuli Selatan, Tahap I, Medan, Balai Arkeologi, 1995. 
-, Laporan Hasil Penelitian Arkeologi Ekskavasi Candi Bara Tahap II/1997, Kabupaten Tapanuli Selatan, Medan, Balai Arkeologi, 1997a.

-, Laporan Hasil Penelitian Arkeologi Ekskavasi Candi Bara Tahap III/1997, Padang Bolak, Kabupaten Tapanuli Selatan, Medan, Balai Arkeologi, $1997 \mathrm{~b}$.

-, Laporan Hasil Penelitian Arkeologi Ekskavasi Candi Bara Tahap IV/1997, Kecamatan Padang Bolak, Kabupaten Tapanuli Selatan, Medan, Balai Arkeologi, 1997c.

Susetyo, Sukawati, «Permukiman di Lingkungan Biaro (Studi Terhadap Biaro Mangaledang, Padang Lawas)», Amerta, 24(1), $2006: 35-41$.

-, et al., Laporan Penelitian Permukinan Kuna di Biaro Naga Saribu, Kompleks Percandian Padang Lawas, Jakarta, Pusat Penelitian Arkeologi, 2003.

Susilowati, Nenggih, "Benteng Tanah, Bidangan, dan Penataan Ruang di Kompleks Kepurbakalaan Si Pamutung, Padang Lawas», Berkala Arkeologi "Sangkhakala" (Medan), 9, $2001: 68-81$.

-, et al., Laporan Penelitian Arkeologi dan Pemetaan Situs Candi Sipamutung dan Sekitarnya, Kecamatan Barumun Tengah, Medan, Balai Arkeologi, 1999.

-, Wiradnyana, K.; Koestoro, L.P., Laporan Penelitian Arkeologi di Tempuran Sungai Barumun dan Batang Pane, Medan, Balai Arkeologi, 2000.

-, Oetomo, R.W.; Sutrisna, D., Laporan Penelitian Arkeologi di Kompleks Kepurbakalaan Sipamutung, Kecamatan Barumun Tengah, Medan, Balai Arkeologi, 2003.

Utomo, Bambang Budi, «Kompleks Percandian Padang Lawas : Sebuah Kompleks Pusat Upacara Agama Buddha Wajrayana», Jurnal Arkeologi Malaysia, 9, 1996 : 60-93.

-, et al., Laporan Penelitian Arkeologi Barumun, Kabupaten Tapanuli Selatan, Sumatera Utara, Tahun 1995, Jakarta, Pusat Penelitian Arkeologi Nasional, 1995.

Zoetmulder, P.J., Kamus Jawa Kuna - Indonesia, Jakarta, GPU/KITLV, 1995 (1 ère éd., 1982). 